\title{
Quotient curves of the Suzuki curve
}

\author{
by \\ Massimo Giulietti (Perugia), Gábor Korchmáros (Potenza) \\ and Fernando Torres (Campinas)
}

1. Introduction. Throughout, let $K=\mathbb{F}_{q}$ be the finite field of order $q$ and $\bar{K}$ its algebraic closure. For a projective, geometrically irreducible, non-singular algebraic curve defined over $K$ (or simply, a curve over $K$ ) of genus $g>0$, the inequality $|\# \mathcal{X}(K)-(q+1)| \leq 2 g \sqrt{q}$ is the Hasse-Weil bound on the number of its $K$-rational points. For possible applications to coding theory [12], [31], [8], correlations of shift register sequences [22], exponential sums [25], or finite geometry [19] one is often interested in those curves with "many rational points". For $q$ a square, the Hermitian curve over $K, Y^{\sqrt{q}} Z+Y Z^{\sqrt{q}}=X^{\sqrt{q}+1}$, attains the Hasse-Weil upper bound; that is to say, it is a maximal curve (see e.g. [31, VI.3.6]).

Let $N_{q}(g)$ denote the maximum number of $K$-rational points on a curve of genus $g$; see Section 7 for further information. A curve over $K$ of genus $g$ whose number of rational points coincides with $N_{q}(g)$ is called optimal. There are three outstanding families of such curves, namely the curves (of positive genus) arising as Deligne-Lusztig varieties of dimension one (DLC); these families are characterized by the following data regarding their number of $K$-rational points, genus and automorphism group over $\bar{K}$ (which actually coincides with the automorphism group over $K$ ); see [5, Sect. 11], [14], [27], [15].

\begin{tabular}{cccc}
\hline Type & $\begin{array}{c}\text { Number of } \\
\text { rational points }\end{array}$ & Genus & $\begin{array}{c}\text { Automorphism } \\
\text { group }\end{array}$ \\
\hline I: $q$ square & $\sqrt{q}^{3}+1$ & $\sqrt{q}(\sqrt{q}-1) / 2$ & $P G U(3, q)$ \\
II: $q=2 q_{0}^{2}>2$ & $q^{2}+1$ & $q_{0}(q-1)$ & $\mathcal{S} z(q)$ \\
III: $q=3 q_{0}^{2}>3$ & $q^{3}+1$ & $3 q_{0}(q-1)\left(q+q_{0}+1\right) / 2$ & $R(q)$ \\
\hline
\end{tabular}

2000 Mathematics Subject Classification: Primary 11G20; Secondary 14G05, 20C33.

Key words and phrases: finite field, Suzuki group, quotient curve, curves with many rational points. 
In addition, the enumerator $L(t)$ of their Zeta function is known (loc. cit.). In the table above $P G U(3, q), \mathcal{S} z(q)$ and $R(q)$ stand for the projective unitary group of degree three, the Suzuki group, and the Ree group over $K$ respectively. The genus, the number of rational points, and the automorphism group of a Hermitian curve coincide with those of a DLC of type I. It turns out that the number of rational points and the genus are the essential data to characterize both Hermitian curves and DLC of type II; see [28], [6, Sect. 3]. (A similar statement for the DLC of type III seems to be unknown.) Motivated by the optimality of a DLC $\mathcal{X}$ and Serre's remark (cf. [21, Prop. 6], [1, Prop. 5]), see Section 3 here, one expects to obtain curves with many rational points from curves $\widetilde{\mathcal{X}}$ that are $K$-covered by $\mathcal{X}$. Having in mind applications it is also important to have such curves in a form as explicit as possible. If $\mathcal{X}$ is the Hermitian curve over $K$, then $L(t)=(\sqrt{q} t+1)^{2 g}$ with $g=\sqrt{q}(\sqrt{q}-1) / 2$; see e.g. [31, V.1.15]. Thus by the aforementioned Serre's remark, the number of $K$-rational points of $\widetilde{\mathcal{X}}$ also attains the Hasse-Weil upper bound. In particular, by means of quotient curves of $\mathcal{X}$, the genus and plane models of a huge number of maximal curves were found; see e.g. [7], [3] and [4].

The aim of this paper is to investigate quotient curves of the DLC of type II; such a curve will be called the Suzuki curve (over $K$ ) and will be denoted by $\mathcal{S}$. The case of curves of type III was investigated by Çakçak and Özbudak [2].

From now on, $q_{0}:=2^{s}$ with $s \geq 1$, and $q:=2 q_{0}^{2}$. The enumerator of the Zeta function of $\mathcal{S}$ is the polynomial $L(t)=\left(q t^{2}+2 q_{0} t+1\right)^{g}$ with $g=q_{0}(q-1)$; see e.g. [14, Prop. 4.3]. Thus the number of $K$-rational points of a curve $\widetilde{\mathcal{S}}$ of genus $\widetilde{g}$ which is $K$-covered by $\mathcal{S}$ is given by (cf. Section 3)

$$
\# \widetilde{\mathcal{S}}(K)=q+2 q_{0} \widetilde{g}+1 .
$$

This value is in the interval from which the entries of the tables of curves with many rational points are taken for $\widetilde{g} \leq 50, q \leq 128$ in van der Geer and van der Vlugt tables [9]. In Sections 4 and 5 we obtain an exhaustive list of tame quotient curves of $\mathcal{S}$, namely quotients arising from subgroups of $\operatorname{Aut}(\mathcal{S})$ of odd order: indeed, we compute the genus as well as exhibit a plane model for such curves. In Section 6 we consider non-tame quotient curves of $\mathcal{S}$, namely quotient arising from subgroups of $\operatorname{Aut}(\mathcal{S})$ of even order; here we cannot produce a complete list as in the odd case because the Suzuki group contains a huge number of pairwise non-isomorphic subgroups of even order. Our contribution consists in proving the existence of non-tame quotient curves of $\mathcal{S}$; for some of these curves we also provide a plane equation. A concrete application of our results provides new 
entries in the tables [9]: let $q=32$ and $r=5$; from Theorems 5.1(2) and $6.10(2)$ we have $N_{32}(24) \geq 225$ and $N_{32}(10) \geq 113$ respectively; cf. Section 7.

The approach employed in this paper is similar to that in [3] and [4]: a concrete realization of $\mathcal{S}$ in $\mathbb{P}^{4}$ is stated via a very ample complete linear series obtained from the enumerator of its Zeta function (cf. Section 3); this embedding is such that $\mathcal{S} z(q)$ acts linearly on $\mathcal{S}$ (cf. Section 2 and Theorem 3.2).

2. Preliminary results on the Suzuki group. In the introduction we have mentioned that the automorphism group $\operatorname{Aut}(\mathcal{S})$ of the Suzuki curve $\mathcal{S}$ is isomorphic to the Suzuki group $\mathcal{S} z(q)$. We summarize those results on the structure of $\mathcal{S} z(q)$ which play a role in the present work. For more details, the reader is referred to [20, Chap. XI.3], [33], [13, Chap. 17] and $[23]$.

In Section 3 we will show that the curve $\mathcal{S}$ can be embedded in $\mathbb{P}^{4}$ (Theorem 3.1); this raises the problem of exhibiting $\mathcal{S} z(q)$ as a subgroup of automorphisms of $\mathbb{P}^{4}$. We start from a well known concrete realization of $\mathcal{S} z(q)$, namely as a subgroup of the automorphism group Aut $\left(\mathbb{P}^{3}\right)$ of $\mathbb{P}^{3}$ (loc. cit.). Let $\widetilde{T}:=\left\{T_{a, c}: a, c \in K\right\}$ and $\widetilde{N}:=\left\{N_{d}: d \in K^{*}\right\}$, where $T_{a, c}$ and $N_{d}$ are the elements of $\operatorname{Aut}\left(\mathbb{P}^{3}\right)$ defined respectively by the matrices

$$
\left(\begin{array}{cccc}
1 & 0 & 0 & 0 \\
a & 1 & 0 & 0 \\
c & a^{2 q_{0}} & 1 & 0 \\
a^{2 q_{0}+2}+a c+c^{2 q_{0}} & a^{2 q_{0}+1}+c & a & 1
\end{array}\right), \quad\left(\begin{array}{cccc}
d^{-q_{0}-1} & 0 & 0 & 0 \\
0 & d^{-q_{0}} & 0 & 0 \\
0 & 0 & d^{q_{0}} & 0 \\
0 & 0 & 0 & d^{q_{0}+1}
\end{array}\right) .
$$

In addition, let $W \in \operatorname{Aut}\left(\mathbb{P}^{3}\right)$ be defined by the matrix

$$
\left(\begin{array}{llll}
0 & 0 & 0 & 1 \\
0 & 0 & 1 & 0 \\
0 & 1 & 0 & 0 \\
1 & 0 & 0 & 0
\end{array}\right)
$$

Then the Suzuki group $\mathcal{S} z(q)$ can be assumed to be the subgroup of $\operatorname{Aut}\left(\mathbb{P}^{3}\right)$ generated by $\widetilde{T}, \widetilde{N}$ and $W$. Next consider the homomorphism of groups

$$
\mathbf{L}: \mathcal{S} z(q) \rightarrow \operatorname{Aut}\left(\mathbb{P}^{4}\right)
$$

defined on the generators of $\mathcal{S} z(q)$ by $T_{a, c} \mapsto \mathbf{T}_{a, c}, N_{d} \mapsto \mathbf{N}_{d}, W \mapsto \mathbf{W}$, where the images are defined respectively by the matrices: 


$$
\begin{aligned}
& \left(\begin{array}{ccccc}
1 & 0 & 0 & 0 & 0 \\
a & 1 & 0 & 0 & 0 \\
a^{q_{0}+1}+c^{q_{0}} & a^{q_{0}} & 1 & 0 & 0 \\
c & a^{2 q_{0}} & 0 & 1 & 0 \\
a^{2 q_{0}+2}+a c+c^{2 q_{0}} & a^{2 q_{0}+1}+c & 0 & a & 1
\end{array}\right) \\
& \left(\begin{array}{ccccc}
d^{-q_{0}-1} & 0 & 0 & 0 & 0 \\
0 & d^{-q_{0}} & 0 & 0 & 0 \\
0 & 0 & 1 & 0 & 0 \\
0 & 0 & 0 & d^{q_{0}} & 0 \\
0 & 0 & 0 & 0 & d^{q_{0}+1}
\end{array}\right), \quad\left(\begin{array}{ccccc}
0 & 0 & 0 & 0 & 1 \\
0 & 0 & 0 & 1 & 0 \\
0 & 0 & 1 & 0 & 0 \\
0 & 1 & 0 & 0 & 0 \\
1 & 0 & 0 & 0 & 0
\end{array}\right) .
\end{aligned}
$$

Let $\widetilde{\mathbf{T}}:=\mathbf{L}(\widetilde{T}), \widetilde{\mathbf{N}}:=\mathbf{L}(\widetilde{N})$ and $\mathbf{W}:=\mathbf{L}(W)$. Then, as $\mathcal{S} z(q)$ is simple (loc. cit.), we can assume that $\mathcal{S} z(q)$ is the subgroup of $\operatorname{Aut}\left(\mathbb{P}^{4}\right)$ generated by $\widetilde{\mathbf{T}}$, $\widetilde{\mathbf{N}}$ and $\mathbf{W}$. Some other basic properties of the Suzuki group that we shall need are the following (loc. cit.):

(2.1) A cyclic subgroup of $\mathcal{S} z(q)$ of order $r>1, r$ a divisor of $q-1$, is conjugate in $\mathcal{S} z(q)$ to the subgroup $\left\{\mathbf{N}_{d} \in \widetilde{\mathbf{N}}: d \in K, d^{r}=1\right\}$.

(2.2) Up to conjugacy there exist two cyclic subgroups of $\mathcal{S} z(q), \mathcal{S}_{1}$ and $\mathcal{S}_{-1}$, of order $q+2 q_{0}+1$ and $q-2 q_{0}+1$ respectively. Such subgroups are called the Singer subgroups of $\mathcal{S} z(q)$. Also, their action on the $K$-rational points of $\pi(\mathcal{S})$, where $\pi$ is the morphism defined in (3.6), is semiregular.

(2.3) The subgroup $\widetilde{\mathbf{T}}$ has exponent 4; that is, the maximum order of its elements is 4 . Its center is an elementary abelian group of order $q$, say $\widetilde{\mathbf{T}}_{2}$, whose elements are those of order 2 of $\widetilde{\mathbf{T}}$. Furthermore, the normalizer of $\widetilde{\mathbf{T}}$ in $\mathcal{S} z(q)$ acts transitively on $\widetilde{\mathbf{T}}_{2}$.

(2.4) The cyclic subgroups of $\mathcal{S} z(q)$ of order 4 are pairwise conjugate in $\mathcal{S} z(q)$.

(2.5) A subgroup of $\mathcal{S} z(q)$ of order $2^{v} r, r, v>1, r$ a divisor of $q-1$, is conjugate in $\mathcal{S} z(q)$ to a subgroup of $\widetilde{\mathbf{T}} \widetilde{\mathbf{N}}$. The order of a non-trivial element in $\widetilde{\mathbf{T}} \widetilde{\mathbf{N}}$ is either a 2-power or a divisor of $q-1$ according as it belongs to $\widetilde{\mathbf{T}}$ or not.

(2.6) A subgroup of $\mathcal{S} z(q)$ of order $2 r, r>1$ a divisor of $q-1$, is conjugate in $\mathcal{S} z(q)$ to a subgroup of $N_{\mathcal{S} z(q)}(\widetilde{\mathbf{N}})$, the normalizer of $\widetilde{\mathbf{N}}$ in $\mathcal{S} z(q)$. The subgroup $N_{\mathcal{S} z(q)}(\tilde{\mathbf{N}})$ is dihedral with $2(q-1)$ elements.

(2.7) A subgroup of $\mathcal{S} z(q)$ of order $2 r, r>1$ a divisor of $q \pm 2 q_{0}+1$, is conjugate in $\mathcal{S} z(q)$ to a subgroup of the (unique) dihedral group of $\mathcal{S} z(q)$ which comprises $\mathcal{S}_{ \pm 1}$. 
(2.8) A subgroup of $\mathcal{S} z(q)$ of order $4 r, r>1$ a divisor of $q \pm 2 q_{0}+1$, is conjugate in $\mathcal{S} z(q)$ to a subgroup of $N_{\mathcal{S} z(q)}\left(\mathcal{S}_{ \pm 1}\right)$, the normalizer of $\mathcal{S}_{ \pm 1}$ in $\mathcal{S} z(q)$. The subgroup $N_{\mathcal{S} z(q)}\left(\mathcal{S}_{ \pm 1}\right)$ has $4\left(q \pm 2 q_{0}+1\right)$ elements. Set $\widetilde{q}:=2^{2 \widetilde{s}+1}$. A necessary and sufficient condition for $\mathcal{S} z(q)$ to contain a subgroup isomorphic to $\mathcal{S} z(\widetilde{q})$ is that $\widetilde{s}$ be a divisor of $s$ such that $2 \widetilde{s}+1$ divides $2 s+1$. For every such divisor, $\mathcal{S} z(q)$ has exactly one conjugacy class of subgroups isomorphic to $\mathcal{S} z(\widetilde{q})$.

Any non-trivial subgroup of $\mathcal{S} z(q)$ is conjugate in $\mathcal{S} z(q)$ to one of the above subgroups.

3. Preliminary results on the Suzuki curve. Let $\mathcal{S}$ be the Suzuki curve over $K\left(q=2 q_{0}^{2}>2\right)$ and $\pi: \mathcal{S} \rightarrow \widetilde{\mathcal{S}}$ a $K$-covering of curves over $K$. Let $L(t)$ and $L_{\widetilde{\mathcal{S}}}(t)$ be the enumerators of the $Z$-function (over $K$ ) of $\mathcal{S}$ and $\widetilde{\mathcal{S}}$ respectively. An observation due to Serre states that $L_{\widetilde{\mathcal{S}}}(t)$ divides $L(t)$ (see [21, Prop. 6], [1, Prop. 5]). As $L(t)=\left(q t^{2}+2 q_{0} t+1\right)^{g}$, where $g$ is the genus of $\mathcal{S}$ (see [14, Prop. 4.3], [27]), we have $L_{\widetilde{\mathcal{S}}}(t)=\left(q t^{2}+2 q_{0} t+1\right)^{\widetilde{g}}$, $\widetilde{g}$ being the genus of $\widetilde{\mathcal{S}}$. In particular, the number of $\mathbb{F}_{q^{r}}$-rational points of $\widetilde{\mathcal{S}}, r \in \mathbb{N}$, can be computed as follows (see e.g. [31, V.1.15]):

$$
\# \widetilde{\mathcal{S}}\left(\mathbb{F}_{q^{r}}\right)=q^{r}+1-\left[(1+i)^{r}+(1-i)^{r}\right]\left(-q_{0}\right)^{r} \widetilde{g},
$$

where $i=\sqrt{-1}$. Notice that $\widetilde{\mathcal{S}}$ is maximal over $\mathbb{F}_{q^{4}}$. Next we describe a plane model of $\mathcal{S}$ which will be the starting point towards the proof of our results. Hansen and Stichtenoth [16] noticed that the number of $K$-rational points and the genus of the non-singular model over $K$ of the plane curve

$$
y^{q}+y=x^{q_{0}}\left(x^{q}+x\right) \quad\left(q=2 q_{0}^{2}>2\right)
$$

are $q^{2}+1$ and $q_{0}(q-1)$ respectively. Therefore by [6, Sect. 3], we may assume that $\mathcal{S}$ is the non-singular model of (3.2). We recall that Henn [18] exhibited this curve as an example of a curve whose number of automorphisms exceeds the Hurwitz upper bound $84(g-1)$ valid in zero characteristic.

Now we introduce a geometric invariant (over $K$ ) on the curve $\mathcal{S}$. Among other properties, this invariant will allow us to consider $\mathcal{S}$ as an embedded curve in $\mathbb{P}^{4}$ in such a way that $\mathcal{S} z(q)$ will act linearly on $\mathcal{S}$; see Theorem 3.2. Let $h(t):=t^{2 g} L\left(t^{-1}\right)$ with $L(t)$ as above. This polynomial is the characteristic polynomial of the Frobenius morphism $\widetilde{\boldsymbol{\Phi}}$ over $K$ on the Jacobian $\mathcal{J}$ of $\mathcal{S}$; this morphism is induced by the Frobenius morphism $\boldsymbol{\Phi}$ over $K$ on $\mathcal{S}$. It turns out that $\widetilde{\boldsymbol{\Phi}}$ is semisimple (see e.g. [26, p. 251]) and thus $q I+2 q_{0} \widetilde{\boldsymbol{\Phi}}+\widetilde{\boldsymbol{\Phi}}^{2}=0$ on $\mathcal{J}$. We can state this property by using divisors on $\mathcal{S}$; to do that we use the fact that $f \circ \mathbf{\Phi}=\widetilde{\boldsymbol{\Phi}} \circ f$, where $f: \mathcal{S} \rightarrow \mathcal{J}$, $P \mapsto\left[P-P_{0}\right]$, is the natural morphism that maps $P_{0} \in \mathcal{S}(K)$ to $0 \in \mathcal{J}$. Therefore, for $P \in \mathcal{S}$ and $P_{0} \in \mathcal{S}(K)$ the following linear equivalence on $\mathcal{S}$ 
holds true:

$$
q P+2 q_{0} \boldsymbol{\Phi}(P)+\boldsymbol{\Phi}^{2}(P) \sim\left(q+2 q_{0}+1\right) P_{0} .
$$

This motivates the definition of the following complete linear series on $\mathcal{S}$ :

$$
\mathcal{D}=\mathcal{D}_{\mathcal{S}}:=\left|\left(q+2 q_{0}+1\right) P_{0}\right| \text {. }
$$

The equivalence (3.3) shows that the definition of $\mathcal{D}$ is independent of the $K$-rational point $P_{0}$, and that $q+2 q_{0}+1$ belongs to the Weierstrass semigroup $H(P)$ at any $K$-rational point $P \in \mathcal{S}$. We subsume two important properties of $\mathcal{D}$ :

\section{THEOREM 3.1.}

(1) ([16, Prop. 1.5]) The dimension of $\mathcal{D}$ is four.

(2) The linear series $\mathcal{D}$ is very ample.

Proof. (1) Let $P \in \mathcal{S}(K)$, and let $i \in \mathbb{N}$ be such that $n_{i}=q+2 q_{0}+1$ $\in H(P)$; by (3.3) we have to show that $i=4$. Let $x, y: \mathcal{S} \rightarrow \mathbb{P}^{1}$ be the $K$-rational functions on $\mathcal{S}$ which define its plane model (3.2). Then $x$ is unramified in $\mathbb{P}^{1}$ but at $\infty$. Over $x=\infty$ there is just one point, say $P_{0} \in \mathcal{S}$, which is, in particular, $K$-rational. It turns out that

$$
\operatorname{div}_{\infty}(x)=q P_{0}, \quad \operatorname{div}_{\infty}(y)=\left(q+q_{0}\right) P_{0} .
$$

Put

$$
z:=x^{2 q_{0}+1}+y^{2 q_{0}} .
$$

By (3.2) the functions $x$ and $z$ satisfy

$$
z^{q}+z=x^{2 q_{0}}\left(x^{q}+x\right)
$$

therefore, $\operatorname{div}_{\infty}(z)=\left(q+2 q_{0}\right) P_{0}$ and so $H\left(P_{0}\right)$ contains the semigroup $H$ generated by $q, q+q_{0}, q+2 q_{0}$ and $q+2 q_{0}+1$. After some computations, one shows that $\#(\mathbb{N} \backslash H)=q_{0}(q-1)$ (see e.g. [16, Appendix]); thus $H\left(P_{0}\right)=H$ and so $i=4$.

(2) Let $\pi: \mathcal{S} \rightarrow \mathbb{P}^{4}$ be the morphism defined by $\mathcal{D}$. We show that $\pi$ separates points and separates tangent vectors; cf. [17, p. 308]. To see the former condition, it is enough to show that $\pi$ is injective. Assume $\pi(P)=$ $\pi(Q)$. Then the linear equivalence (3.3) implies

$$
\left\{P, \boldsymbol{\Phi}(P), \boldsymbol{\Phi}^{2}(P)\right\}=\left\{Q, \boldsymbol{\Phi}(Q), \boldsymbol{\Phi}^{2}(Q)\right\} .
$$

We find that $\boldsymbol{\Phi}^{3}(P)=P$ and $\boldsymbol{\Phi}^{3}(Q)=Q$; thus $P=Q$ since $\mathcal{S}\left(\mathbb{F}_{q^{3}}\right)=\mathcal{S}(K)$ by (3.1). To prove the latter condition we use some facts concerning Weierstrass point theory; our reference is Stöhr-Voloch's paper [32]. We have to show that the first positive element $j_{1}=j_{1}(P)$ of the $(\mathcal{D}, P)$-order sequence equals 1 , or equivalently that there exists $D^{\prime} \in \mathcal{D}$ such that $v_{P}\left(D^{\prime}\right)=1$. If $P \notin \mathcal{S}(K)$, then $j_{1}=1$ by (3.1) and (3.3); otherwise, let $n_{1}<n_{2}<n_{3}<n_{4}=q+2 q_{0}+1$ be the first four positive elements of $H(P)$. 
Then $j_{1}=n_{4}-n_{3}$, and it is enough to show that $n_{3}=q+2 q_{0}(*)$. (Observe that $(*)$ already holds true for the point $P_{0}$ over $x=\infty$.) As a matter of fact, property $(*)$ was proved in $[6$, p. 43$]$ and the proof of the theorem is complete.

In order to study the concrete realization of $\mathcal{S}$ in $\mathbb{P}^{4}$ we use the $K$-rational morphism

$$
\pi:=(1: x: y: z: w)
$$

where $x, y, z$ are as above and $w$ is a $K$-rational function such that $\operatorname{div}_{\infty}(w)$ $=\left(q+2 q_{0}+1\right) P_{0}$. We may assume

$$
w:=x y^{2 q_{0}}+z^{2 q_{0}} .
$$

In fact, (3.2) and (3.5) imply the following relation among $x, y$ and $w$ :

$$
w^{q}+w=y^{2 q_{0}}\left(x^{q}+x\right),
$$

whence $\operatorname{div}_{\infty}(w)=\left(q+2 q_{0}+1\right) P_{0}$. Next we point out some relations describing $y, w$ as functions depending on $x$ and $z$ only. By raising (3.4) to the power $q_{0}$ and using (3.2) we obtain

$$
y=x^{q_{0}+1}+z^{q_{0}}
$$

from (3.7), (3.8) and (3.5) we get

$$
w=x^{2 q_{0}+2}+x z+z^{2 q_{0}} .
$$

Therefore $\mathcal{S}$ can be assumed to be the locus in $\mathbb{P}^{4}$ defined by the set of points

$$
P_{(a, c)}:=(1: a: b: c: d), \quad \text { and } \quad A_{4}:=\pi\left(P_{0}\right)=(0: 0: 0: 0: 1) \text {, }
$$

with $x=a \in \bar{K}, z=c \in \bar{K}$ satisfying (3.5), and $y=b$ and $w=d$ defined by (3.8) and (3.9) respectively.

Now we show that the concrete realization of the Suzuki group $\mathcal{S} z(q)$ established in Section 2 coincides with $\operatorname{Aut}(\mathcal{S})$.

Theorem 3.2. The isomorphic image of $\mathcal{S} z(q)$ in $\operatorname{Aut}\left(\mathbb{P}^{4}\right)$ stated in Section 2 acts linearly on $\mathcal{S}$.

Proof. By (3.10) and the definition of $\widetilde{\mathbf{T}}, \widetilde{\mathbf{N}}$ and $\mathbf{W}$ (cf. Section 2) it follows that each element of the isomorphic image of $\mathcal{S} z(q)$ in $\operatorname{Aut}\left(\mathbb{P}^{4}\right)$ acts linearly on $\mathcal{S}$.

REMARK 3.3. If in (3.10) we only consider the points corresponding to $a, c \in K$ together with the point $A_{4}$, we obtain the so-called Suzuki-Tits ovoid (cf. [33]); this was already noticed by Cossidente [6, Appendix].

\section{Quotient curves arising from subgroups of a cyclic subgroup} of $\operatorname{Aut}(\mathcal{S})$ of order $q-1$. For a divisor $r>1$ of $q-1$, let $\mathcal{U}$ be a cyclic subgroup of $\operatorname{Aut}(\mathcal{S})$ of order $r$. As mentioned in $(2.1), \mathcal{U}$ is unique up to conjugacy, and we may assume $\mathcal{U}=\left\{\mathbf{N}_{d} \in \widetilde{\mathbf{N}}: d \in K, d^{r}=1\right\}$. Let $\widetilde{\mathcal{S}}=\mathcal{S} / \mathcal{U}$ 
denote the quotient curve of $\mathcal{S}$ by $\mathcal{U}$, and $\widetilde{g}$ its genus. The objective of this section is to prove the following theorem. Let $s \in \mathbb{N}$ be such that $q_{0}=2^{s}$, and set

$$
f(t):=1+\sum_{i=0}^{s-1} t^{2^{i}\left(2 q_{0}+1\right)-\left(q_{0}+1\right)}(1+t)^{2^{i}} .
$$

TheOREM 4.1. With the notation above:

(1) $\widetilde{g}=\frac{1}{r} q_{0}(q-1)$.

(2) The following curve is a plane model over $K$ of $\widetilde{\mathcal{S}}$ :

$$
V^{\frac{1}{r}(q-1)} f(U)=\left(1+U^{q_{0}}\right)\left(U^{q-1}+V^{\frac{2}{r}(q-1)}\right) .
$$

We need some preliminary results. Consider the morphism

$$
\phi:=(x: y: z): \mathcal{S} \rightarrow \mathcal{C}:=\phi(\mathcal{S}) \subseteq \mathbb{P}^{2} .
$$

The results in Claims 4.2-4.4 and Lemma 4.5 below will show that $\mathcal{C}$ is a plane model over $K$ of $\mathcal{S}$.

Claim 4.2.

(1) The divisor defined by $\phi$ is given by $E=-A_{0}+\left(q+2 q_{0}\right) A_{4}$.

(2) The morphism $\phi$ is birational so that $\mathcal{C}$ is a curve of degree $q+2 q_{0}-1$.

Proof. (1) By (3.2), (3.5) and the fact that $x: \mathcal{S} \rightarrow \mathbb{P}^{1}$ is unramified but at $\infty$, where it is totally ramified,

$$
\begin{aligned}
& \operatorname{div}(x)=A_{0}+D_{1}-q A_{4}, \\
& \operatorname{div}(y)=\left(q_{0}+1\right) A_{0}+D_{2}-\left(q+q_{0}\right) A_{4}, \\
& \operatorname{div}(z)=\left(2 q_{0}+1\right) A_{0}+D_{3}-\left(q+2 q_{0}\right) A_{4},
\end{aligned}
$$

with $D_{1}=\sum P_{\left(0, y^{2 q_{0}}\right)}, y^{q-1}=1 ; D_{2}=\sum P_{\left(x, x^{2 q_{0}+1}\right)}, x^{q-1}=1$; and $D_{3}=$ $\sum P_{(x, 0)}, x^{q-1}=1$. Therefore $E=-A_{0}+\left(q+2 q_{0}\right) A_{4}$.

(2) For a generic point $P_{(a, c)} \in \mathcal{S}$ we show $\# \phi^{-1}\left(\phi\left(P_{(a, c)}\right)\right)=1$. Let $(x / y, z / y)=(a / b, c / b)$. By $(3.8)$,

$$
a^{q_{0}+1} y^{q_{0}}+b c^{q_{0}} y^{q_{0}-1}+b^{q_{0}+1}=0,
$$

and after some computations one realizes that $y=b$ is the only solution of this equation such that $P_{(x, z)} \in \mathcal{S}$.

Let $(X: Y: Z)$ be projective coordinates for $\mathbb{P}^{2}$ and assume that $Y=0$ is the line at infinity. We look for the equation $f_{1}(X, Z)=0$ that defines the plane curve $\mathcal{C}$ above. The intersection divisor of $Y$ and $\mathcal{C}$ is codified by the divisor $\phi^{*}(Y):=\operatorname{div}(y)+E=q_{0} A_{0}+D_{2}+q_{0} A_{4}$; cf. Claim 4.2 and (4.2). This means that the line $Y$ intersects $\mathcal{C}$ at $q+1$ points, $q_{0}$ being the order of contact at both $\phi\left(A_{0}\right)$ and $\phi\left(A_{4}\right)$. Thus the term of degree $q+2 q_{0}-1$ of 
$f_{1}(X, Z)$ can be assumed to be $(X Z)^{q_{0}}\left(X^{q-1}+Z^{q-1}\right)$ and hence the defining equation of $\mathcal{C}$ will be of type

$$
f_{1}(X, Z)=f_{2}(X, Z)+\left(f_{3}(X, Z)+(X Z)^{q_{0}}\right)\left(X^{q-1}+Z^{q-1}\right),
$$

with $\operatorname{deg}\left(f_{2}(X, Z)\right)<q+2 q_{0}-1$ and $\operatorname{deg}\left(f_{3}(X, Z)\right)<2 q_{0}$. Now each $\mathbf{N}_{d} \in \widetilde{\mathbf{N}}$ induces an automorphism on $\mathcal{C}$ by means of $(X: Y: Z) \mapsto\left(d X: d^{q_{0}+1} Y\right.$ : $\left.d^{2 q_{0}+1} Z\right)$. Thus there exists $e \in \bar{K}^{*}$ such that $f_{1}(X, Z)=e f_{1}\left(d^{-q_{0}} X, d^{q_{0}} Z\right)$; we then have $e=1$ by looking at the higher degree term of $f_{1}(X, Z)$. Furthermore, $f_{2}(X, Z)=\sum_{i, j} a_{i, j} X^{i} Z^{j}, a_{i, j}=a_{i, j} d^{j-i}$. Suppose that $a_{i, j} \neq 0$, so that $d^{j-i}=1$. Therefore $q-1$ divides $j-i$ whenever $d$ is a primitive element of $K^{*}$; thus either $j=i, j=i+q-1$, or $i=j+q-1$. Write

$$
f_{1}(X, Z)=f_{2}(X, Z)+X^{q-1} \sum_{i, j ; i \neq j} a_{i, j}(X Z)^{j}+Z^{q-1} \sum_{i, j ; i \neq j} a_{i, j}(X Z)^{i} .
$$

On the other hand, the automorphism $\mathbf{W}$ of $\mathcal{S}$ (cf. Section 2) induces an automorphism on $\mathcal{C}$ via $(X: Y: Z) \mapsto(Z: Y: X)$. Thus $a_{i, j}=a_{j, i}$ and so

$$
f_{1}(X, Z)=\widetilde{f}_{2}(X Z)+\left(\widetilde{f}_{3}(X Z)+(X Z)^{q_{0}}\right)\left(X^{q-1}+Z^{q-1}\right),
$$

where $\widetilde{f}_{2}(t)$ and $\widetilde{f}_{3}(t)$ are polynomials of degree at most $q / 2+q_{0}-1$ and $q_{0}-1$ respectively.

Claim 4.3. $\operatorname{deg}\left(\widetilde{f}_{2}(t)\right)=(q-2) / 2$.

Proof. For the line $L: X+Z=0$, we compute $\phi^{*}(L)$ which is equal to $\operatorname{div}_{0}(x+z)-A_{0}$ by Claim 4.2(1). Let $P:=P_{(a, a)}$ be a zero of $x+z$ so that

$$
\begin{aligned}
(x+z)^{q}+(x+z)= & (x+a)^{q+2 q_{0}}+\left(a^{2 q_{0}}+1\right)(x+a)^{q}+(x+a)^{2 q_{0}+1} \\
& +\left(a^{q}+a\right)(x+a)^{2 q_{0}}+\left(a^{2 q_{0}}+1\right)(x+a) .
\end{aligned}
$$

Since $x+a$ is a local parameter at $P$, the valuation of $x+z$ at $P$ is equal to either $2 q_{0}+1$ or 1 according as $a=1$ or $a \in K \backslash\{1\}$. Thus

$$
\phi^{*}(L)=\left(2 q_{0}+1\right) P_{(1,1)}+\sum_{x \notin K \backslash\{0,1\}} P_{(x, x)}
$$

and hence $\operatorname{deg}\left(\widetilde{f}_{2}\left(t^{2}\right)\right)=q-2$ as $\phi\left(P_{(1,1)}\right)$ belongs to the line at infinity.

Next we determine the explicit expression for $\widetilde{f}_{2}(t)$ and $\widetilde{f}_{3}(t)$, namely we show that $\widetilde{f}_{2}(t)=f(t)$ and $\widetilde{f}_{3}(t)=1$.

Claim 4.4 .

(1) There exists e $\in K^{*}$ such that ef $(t)=\widetilde{f}_{2}(t)$, where $f(t)$ is the polynomial defined in (4.1).

(2) $\widetilde{f}_{3}(t)=1$ and $e=1$. 
Proof. (1) We shall show that $f(t)$ and $\widetilde{f}_{2}(t)$ have $(q-2) / 2$ common roots in $K$. Let $a \in K$; after some computations we have

$$
\left(a^{2 q_{0}-1}\right)^{q_{0}} f\left(a^{2 q_{0}-1}\right)=\operatorname{Tr}_{K \mid \mathbb{F}_{2}}(a)
$$

Since the map $a \mapsto a^{2 q_{0}-1}$ is a bijection on $K$, the set of roots of $f(t)$ consists precisely of the $(q-2) / 2$ elements of the set

$$
\left\{a^{2 q_{0}-1}: \operatorname{Tr}_{K \mid \mathbb{F}_{2}}(a)=0, a \neq 0\right\} \text {. }
$$

Now by $(*)$ this set is invariant by the quadratic map $a \mapsto a^{2}$, and hence their elements are precisely the roots of $\widetilde{f}_{2}(t)$.

(2) By (1), we have

$$
e f(\xi \zeta)=\left(\tilde{f}_{3}(\xi \zeta)+(\xi \zeta)^{q_{0}}\right)\left(\xi^{q_{0}}+\zeta^{q_{0}}\right)
$$

where $\xi:=x / y$ and $\zeta:=z / y$. We use local power series computations at the point $A_{0}$, where $x$ is a local parameter; by dots we mean terms of higher degree. From (3.2) and (3.5), $\xi=x^{-q_{0}}+x^{q-q_{0}+1}+\cdots$ and $\zeta=x^{q_{0}}+x^{q^{\prime}}+\cdots$, where $q^{\prime}>q+q_{0}-1$; thus $\xi \zeta=1+x^{q-1}+\cdots$. By the definition of $f(t)$, $f=1+x^{q-1}+\cdots$. Write $\tilde{f}_{3}(t)=a_{0}+a_{m} t^{m}+a_{m+1} t^{m+1}+\cdots$, and suppose that $m=2^{n} k \geq 1$ and $a_{m} \neq 0$. Then $\widetilde{f}_{3}=a_{0}+a_{m}\left(1+x^{q-1}+\cdots\right)^{2 n}+\cdots$, and by comparing powers of $x$ (via $(* *))$ we must have $(q-1) 2^{n}-q_{0}(q-1)=0$, which is a contradiction as $2^{n}<q_{0}$. Thus $(* *)$ becomes

$$
e\left(1+x^{q-1}+\cdots\right)=e f(\xi \zeta)=\left(a_{0}+1+x^{(q-1) q_{0}}+\cdots\right)\left(x^{-(q-1) q_{0}}+\cdots\right),
$$

and so $a_{0}=1$ and $e=1$.

We summarize the results above in the following.

LEMMA 4.5. The Suzuki curve $\mathcal{S}$ admits a plane model over $K$ defined by

$$
f_{1}(X, Z):=f(X Z)+\left(1+(X Z)^{q_{0}}\right)\left(X^{q-1}+Z^{q-1}\right),
$$

where $f(t)$ is the polynomial given in (4.1).

Proof of Theorem 4.1. The genus $\widetilde{g}$ : It is straightforward to check that $\mathcal{U}$ has exactly two fixed points on $\mathcal{S}$, namely $A_{0}:=(1: 0: 0: 0: 0)$ and $A_{4}=(0: 0: 0: 0: 1)$. Since $\widetilde{\mathcal{S}}$ is a tame quotient curve, the RiemannHurwitz genus formula gives $2 q_{0}(q-1)-2=r(2 \widetilde{g}-2)+2(r-1)$, whence the result follows.

The plane equation: Let $\widetilde{\mathcal{C}}$ be the absolutely irreducible plane curve over $K$ whose function field is $K\left(X Z, Z^{r}\right)$. By the definition of $\mathbf{N}_{d}$, we have the inclusion $K\left(X Z, Z^{r}\right) \subseteq K(X, Z)^{\mathcal{U}}$. On the other hand, from the definition of $f_{1}(X, Z)$ in Lemma 4.5 ,

$$
\left[K(X, Z): K\left(X Z, Z^{r}\right)\right]=\left[K(X Z, Z): K\left(X Z, Z^{r}\right)\right]=r
$$

and thus the above inclusion is an equality. Taking $U:=X Z$ and $V:=Z^{r}$ we obtain the required equation for $\widetilde{\mathcal{C}}$, which is clearly a plane model of $\widetilde{\mathcal{S}}$. 
REMARK 4.6. The geometric meaning of the proof of Lemma 4.5 emerges from the fact that the automorphisms $\mathbf{N}_{d}$ and $\mathbf{W}$ of $\mathcal{S}$ preserve the line $L$ in $\mathbb{P}^{4}$ that joins the points $A_{0}$ and $A_{4}$. Indeed, the morphism $\phi$ above has been chosen to be the morphism associated to the 2-dimensional linear series cut out on $\mathcal{S}$ by hyperplanes through $L$.

REMARK 4.7. The curve $\widetilde{\mathcal{S}}$ in Theorem 4.1 is a covering of the hyperelliptic curve $v f(u)=\left(1+u^{q_{0}}\right)\left(u^{q-1}+v^{2}\right)$ of genus $\widetilde{q}_{0}$; upon replacing $v$ by $\left(1+u^{q_{0}}\right) v / f(u)$, such a hyperelliptic curve can be defined by

$$
v^{2}+v=\frac{(u+1)^{2 q_{0}} u^{q-1}}{f(u)^{2}} .
$$

5. Quotient curves arising from the subgroups of the Singer subgroups of $\operatorname{Aut}(\mathcal{S})$. Let $\mathcal{S}_{1}$ and $\mathcal{S}_{-1}$ denote the Singer subgroups of $\operatorname{Aut}(\mathcal{S})$ whose orders are $q+2 q_{0}+1$ and $q-2 q_{0}+1$ respectively; cf. (2.2). For a subgroup $\mathcal{U}$ of either $\mathcal{S}_{1}$ or $\mathcal{S}_{-1}$, denote by $\widetilde{\mathcal{S}}=\mathcal{S} / \mathcal{U}$ the quotient curve of $\mathcal{S}$ by $\mathcal{U}$, and $\widetilde{g}$ its genus. The aim of this section is to prove the following theorem. Let $s \in \mathbb{N}$ be such that $q_{0}=2^{s}$, and set

$$
\widetilde{f}(t):=1+\sum_{i=0}^{s-1} t^{2^{i} q_{0}}(1+t)^{2^{i}\left(q_{0}+1\right)-q_{0}}+t^{q / 2} .
$$

THEOREM 5.1. With the notation above:

(1) If $\mathcal{U}$ is a subgroup of $\mathcal{S}_{1}$ of order $r>1$, then

$$
\widetilde{g}=\frac{1}{r}\left(q+2 q_{0}+1\right)\left(q_{0}-1\right)+1
$$

and a plane model over $\mathbb{F}_{q^{4}}$ of $\widetilde{\mathcal{S}}$ is given by

$$
V^{\frac{1}{r}\left(q+2 q_{0}+1\right)} \tilde{f}(U)=U^{q+2 q_{0}+1}+V^{\frac{2}{r}\left(q+2 q_{0}+1\right)} .
$$

(2) If $\mathcal{U}$ is a subgroup of $\mathcal{S}_{-1}$ of order $r>1$, then

$$
\widetilde{g}=\frac{1}{r}\left(q-2 q_{0}+1\right)\left(q_{0}+1\right)-1
$$

and a plane model over $\mathbb{F}_{q^{4}}$ of $\widetilde{\mathcal{S}}$ is given by

$$
b V^{\frac{1}{r}\left(q-2 q_{0}+1\right)} f(U)=\left(U^{q_{0}-1}+U^{2 q_{0}-1}\right)\left(U^{q-2 q_{0}+1}+V^{\frac{2}{r}\left(q-2 q_{0}+1\right)}\right),
$$

where $\widetilde{f}(t)$ and $f(t)$ are the polynomials defined in (5.1) and (4.1) respectively, and $b:=\lambda^{q_{0}}+\lambda^{q_{0}-1}+\lambda^{-q_{0}}+\lambda^{-\left(q_{0}-1\right)}$ with $\lambda \in \mathbb{F}_{q^{4}}$ of order $q-2 q_{0}+1$. 
To work out a plane model for $\widetilde{\mathcal{S}}$, we use the same approach as in Section 4. In particular, we write out appropriate plane models of $\mathcal{S}$; see Lemmas 5.5 and 5.7 below. We obtain these results through Claims 5.2-5.4 and Claim 5.6 respectively.

To begin with, we look for a suitable birational morphism

$$
\phi=\left(h_{0}: h_{1}: h_{2}\right): \mathcal{S} \rightarrow \mathcal{C}:=\phi(\mathcal{S}) \subseteq \mathbb{P}^{2}
$$

of degree $q+2 q_{0}+1$ or $q+2 q_{0}-1$ with $h_{0}, h_{1}, h_{2} \in \mathcal{L}\left(\left(q+2 q_{0}+1\right) P_{0}\right)=$ $\langle 1, x, y, z, w\rangle$, where $x, y, z$ and $w$ are the rational functions on $\mathcal{S}$ defined in (3.2), (3.4) and (3.7) respectively. Let $\lambda \in \mathbb{F}_{q^{4}}$ be an element of order $q \pm 2 q_{0}+1$ and set

$$
b:= \begin{cases}\lambda^{q_{0}}+\lambda^{q_{0}+1}+\lambda^{-q_{0}}+\lambda^{-\left(q_{0}+1\right)} & \text { if } \lambda \text { has order } q+2 q_{0}+1, \\ \lambda^{q_{0}}+\lambda^{q_{0}-1}+\lambda^{-q_{0}}+\lambda^{-\left(q_{0}-1\right)} & \text { if } \lambda \text { has order } q-2 q_{0}+1 .\end{cases}
$$

Notice that $b^{q-1}=1$. Let

$$
\mu:=\frac{\lambda+\lambda^{-1}}{b},
$$

so that $\mu^{q^{2}-1}=1$. Choose $h_{1}$ and $h_{2}$ with the following properties:

- $v_{A_{4}}\left(h_{1}\right)=v_{A_{4}}\left(h_{2}\right)=-\left(q+2 q_{0}+1\right)$.

- Let $P=P_{(a, c)} \in \mathcal{S}$. Then $a=\mu$ whenever $h_{1}(P)=h_{2}(P)=0$.

Set

$$
h_{0}:=b^{q_{0}-1} x+y+b^{q_{0}-1}
$$

in such a way that $\phi$ is birational; cf. (5.5) below. Let $E$ be the divisor defined by $\phi$. Then $v_{A_{4}}(E)=q+2 q_{0}+1$, and the base points of the linear series associated to $\phi$ are the common zeroes of $h_{0}, h_{1}$ and $h_{2}$.

Claim 5.2. We have

$$
E= \begin{cases}\left(q+2 q_{0}+1\right) A_{4} & \text { if } \lambda^{q+2 q_{0}+1}=1, \\ -P_{\left(\mu, \mu \lambda^{q}\right)}-P_{\left(\mu, \mu \lambda^{q}+b\right)}+\left(q+2 q_{0}+1\right) A_{4} & \text { if } \lambda^{q-2 q_{0}+1}=1 .\end{cases}
$$

In particular, the plane curve $\mathcal{C}$ above has degree $q+2 q_{0}+1$ or $q+2 q_{0}-1$.

Proof. Let $P_{(a, c)} \in \mathcal{S}$ be a common zero of $h_{0}, h_{1}$ and $h_{2}$ with $a=\mu$ and $c \in \bar{K}$ defined via (3.5). If $\widetilde{c}:=c / b$, then $b\left(\widetilde{c}^{q}+\widetilde{c}\right)=\mu^{2 q_{0}}\left(\mu^{q}+\mu\right)$; now taking into consideration (3.8) and the definition of $h_{0}$, we conclude that $\widetilde{c}$ is a solution of the system

$$
b^{q_{0}} T^{q_{0}}+b^{q_{0}} T=b^{q_{0}-1} \mu+\mu^{q_{0}+1}, \quad b\left(T^{q}+T\right)=\mu^{2 q_{0}}\left(\mu^{q}+\mu\right) .
$$

From the definition of $b$ it follows that $\widetilde{c}$ must be a root of the quadratic equation

$$
T^{2}+T=(\mu / b)^{2}
$$


It turns out that the solutions of this equation are $t:=\mu \lambda^{q} / b$ and $t+1$ since

$$
\lambda^{2 q}+b^{2} \frac{\lambda^{q}}{\lambda+\lambda^{-1}}+1=0 .
$$

It is straightforward to check that $t$ and $t+1$ satisfy (5.2) if and only if $\lambda^{q-2 q_{0}+1}=1$, and the proof is complete.

Assume that $\lambda$ has order $q+2 q_{0}+1$. Let $(X: Y: Z)$ be projective coordinates of $\mathbb{P}^{2}$ and assume that $X=0$ is the line at infinity. We look for an equation $f_{1}(Y, Z)=0$ for the plane curve $\mathcal{C}$. The intersection divisor of $X$ and $\mathcal{C}$ is codified by $\phi^{*}(X)=\operatorname{div}\left(h_{0}\right)+E=\operatorname{div}_{0}\left(h_{0}\right)+A_{4}$ by the previous claim. Let $\mathbf{B}:=\mathbf{T}_{0, b} \circ \mathbf{W} \in \operatorname{Aut}(\mathcal{S})$. Thus $\mathbf{B}$ is defined by the matrix (cf. Section 2)

$$
B=\left(\begin{array}{ccccc}
0 & 0 & 0 & 0 & 1 \\
0 & 0 & 0 & 1 & 0 \\
0 & 0 & 1 & 0 & b^{q_{0}} \\
0 & 1 & 0 & 0 & b \\
1 & 0 & 0 & b & b^{2 q_{0}}
\end{array}\right) .
$$

We shall prove that the points $\mathbf{B}^{i}\left(A_{0}\right)\left(i=2, \ldots, q+2 q_{0}+1\right)$ are zeroes of $h_{0}$. To see this, we apply induction on $i$; indeed, it is enough to show that $h_{0}\left(\mathbf{B}^{2}\left(A_{0}\right)\right)=0$, which is a straightforward computation. On the other hand, $\mathbf{B}\left(A_{0}\right)=A_{4}$ and so the aforementioned points are precisely the zeroes of $h_{0}$ (recall that $\operatorname{deg}\left(h_{0}\right)=q+2 q_{0}$ ); thus

$$
\phi^{*}(X)=\sum_{i=1}^{q+2 q_{0}+1} \mathbf{B}^{i}\left(A_{0}\right) .
$$

To see the significance of this computations on $\mathcal{C}$ we have to compute $\phi\left(\mathbf{B}^{i}\left(A_{0}\right)\right), i=1, \ldots, q+2 q_{0}+1$. At this point we choose concrete rational functions $h_{1}$ and $h_{2}$ on $\mathcal{S}$. Let $\lambda \in \mathbb{F}_{q^{4}}$ be of order $q+2 q_{0}+1$ and $\mathbf{M}$ the automorphism of $\mathbb{P}^{4}$ defined by the matrix

$$
M:=\left(\begin{array}{ccccc}
0 & b^{q_{0}-1} & 1 & b^{q_{0}-1} & 0 \\
\mu & 1 & 0 & \lambda & \lambda \mu \\
\mu^{q} & 1 & 0 & \lambda^{q} & \lambda^{q} \mu^{q} \\
\mu & 1 & 0 & \lambda^{-1} & \lambda^{-1} \mu \\
\mu^{q} & 1 & 0 & \lambda^{-q} & \lambda^{-q} \mu^{q}
\end{array}\right) .
$$

A straightforward computation shows that

$$
M B M^{-1}=\Lambda \text {, }
$$


where

$$
\Lambda:=\left(\begin{array}{ccccc}
1 & 0 & 0 & 0 & 0 \\
0 & \lambda & 0 & 0 & 0 \\
0 & 0 & \lambda^{q} & 0 & 0 \\
0 & 0 & 0 & \lambda^{-1} & 0 \\
0 & 0 & 0 & 0 & \lambda^{-q}
\end{array}\right) .
$$

We observe that the first row of $M$ defines $h_{0}$, and we now define $h_{1}$ and $h_{2}$ by using the second and fourth row respectively; that is to say,

$$
h_{1}:=\mu+x+\lambda z+\lambda \mu w, \quad h_{2}:=\mu+x+\lambda^{-1} z+\lambda^{-1} \mu w .
$$

In particular, the Suzuki curve is birationally equivalent over $\mathbb{F}_{q^{4}}$ to $\mathcal{C}=\phi(\mathcal{S})$ since $\mathbf{M} \in \operatorname{Aut}\left(\mathbb{P}^{4}\right)$ is defined over $\mathbb{F}_{q^{4}}$. From (5.4),

$$
\mathbf{B}^{i}\left(A_{0}\right)=\mathbf{M}^{-1}\left(0: \lambda^{i} \mu: \lambda^{i q} \mu^{q}: \lambda^{-i} \mu: \lambda^{-i q} \mu^{q}\right)
$$

and hence $\phi\left(\mathbf{B}^{i}\left(A_{0}\right)\right)=\left(0: \lambda^{i}: \lambda^{-i}\right), i=1, \ldots, q+2 q_{0}+1$. Thus we may assume that $\mathcal{C}$ is defined by

$$
f_{1}(Y, Z)=f_{2}(Y, Z)+\left(Y^{q+2 q_{0}+1}+Z^{q+2 q_{0}+1}\right),
$$

$f_{2}(Y, Z)$ being a polynomial of degree at most $q+2 q_{0}$. To normalize $f_{2}(Y, Z)$ we use the fact that the automorphism $\mathbf{B}$ above induces an automorphism on $\mathcal{C}$ via $(X: Y: Z) \mapsto\left(X: \lambda^{-1} Y: \lambda Z\right)$; then we proceed as in the proof of Lemma 4.5 to conclude that

$$
f_{2}(Y, Z)=\widetilde{f}_{2}(Y Z)
$$

where $\widetilde{f}_{2}(t)$ is a polynomial of degree less than $\left(q+2 q_{0}+1\right) / 2$.

Claim 5.3. $\operatorname{deg}\left(\widetilde{f}_{2}(t)\right)=q / 2$.

Proof. Consider the line $L: Y+\lambda^{2} Z=0$; since $\lambda^{2} h_{2}+h_{1}=\left(\lambda^{2}+1\right)(x+\mu)$,

$$
\phi^{*}(L)=\operatorname{div}(x+\mu)+E=\operatorname{div}_{0}(x+\mu)+\left(2 q_{0}+1\right) A_{4}
$$

(cf. (4.2) and Claim 5.2). Thus $\operatorname{deg}\left(\widetilde{f}_{2}\left(t^{2}\right)\right)=q$ as $\phi\left(A_{4}\right) \in X$.

Claim 5.4. The polynomial $\widetilde{f}(t)$ in (5.1) coincides with $\widetilde{f}_{2}(t)$.

Proof. We show that both polynomials $\widetilde{f}(t)$ and $\widetilde{f}_{2}(t)$ have $q / 2$ different common roots. After some computations, for $a \in K$,

$$
\left(1+a^{2 q_{0}-1}\right)^{q_{0}} \widetilde{f}\left(a^{2 q_{0}-1}\right)=1+\operatorname{Tr}_{K \mid \mathbb{F}_{2}}(a) ;
$$

thus the set of roots of $\widetilde{f}(t)$ is given by

$$
\left\{a^{2 q_{0}-1}: a \in K, \operatorname{Tr}_{K \mid \mathbb{F}_{2}}(a)=1\right\} .
$$


Now we compute the roots of $\widetilde{f}_{2}(t)$. By the proof of Claim 5.3, such roots arise from the points $(1: Y: Z) \in \mathcal{C}$ with $Y=\lambda^{2} Z$. We have

$$
(1: Y: Z)=\left(1: \lambda B(z)^{2 q_{0}-1}: \lambda^{-1} B(z)^{2 q_{0}-1}\right),
$$

where

$$
B(z):=z^{q_{0}}+z+\frac{\mu}{b}+\frac{\mu^{q_{0}+1}}{b^{q_{0}}}
$$

with $z$ such that $b\left(z^{q}+z\right)=\mu^{2 q_{0}}\left(\mu^{q}+\mu\right)(*)$. Therefore $a \in \bar{K}$ is a root of $\widetilde{f}_{2}(t)$ if and only if $a=B(z)^{4 q_{0}-2}, z$ being as in $(*)$. Now $B(z) \in K$, and by using the fact that the map $a \mapsto a^{2 q_{0}-1}$ is a bijection on $K$, and that the set of roots of $\widetilde{f}(t)$ is invariant under the map $b \mapsto b^{2}$, we conclude that there exists $e \in \bar{K}$ such that $\widetilde{f}(t)=e \widetilde{f}_{2}(t)$. Now $\phi\left(P_{\left(\mu^{-1}, \lambda \mu^{-1}\right)}\right)=\left(1: 0: \lambda^{-1}\right)$ and we have $e=1$.

We have thus proved the following lemma.

Lemma 5.5. The Suzuki curve $\mathcal{S}$ admits a plane model over $\mathbb{F}_{q^{4}}$ defined by

$$
f_{1}(Y, Z)=\tilde{f}(Y Z)+\left(Y^{q+2 q_{0}+1}+Z^{q+2 q_{0}+1}\right)
$$

where $\tilde{f}(t)$ is as in (5.1).

Now let $\lambda \in \mathbb{F}_{q^{4}}$ be of order $q-2 q_{0}+1$. We compute the intersection divisor of $X$ and $\mathcal{C}$. By (4.2) and Claim 5.2,

$$
\phi^{*}(X)=\operatorname{div}\left(h_{0}\right)+E=\operatorname{div}_{0}\left(h_{0}\right)-P_{1}-P_{2}+A_{4},
$$

where $P_{1}:=P_{\left(\mu, \lambda^{q} \mu\right)}$ and $P_{2}:=P_{\left(\mu, \lambda^{q} \mu+b\right)}$; by arguing as in the proof of the previous lemma, we check that the points $\mathbf{B}^{i}\left(A_{0}\right)\left(i=2, \ldots, q-2 q_{0}+1\right)$ are zeroes of $h_{0}$; thus, up to multiplicity, there are $4 q_{0}$ zeroes of $h_{0}$ missing. By the definition of $h_{0}$ and equations (3.2) and (3.5) we have

$$
\begin{aligned}
h_{0}^{q}+h_{0}= & \left(x^{q}+x\right)\left(b^{q_{0}-1}+x^{q_{0}}+b^{q_{0}-1} x^{2 q_{0}}\right) \\
= & {\left[(x+a)^{q}+(x+a)+a^{q}+a\right]\left[(x-a)^{q_{0}}+b^{q_{0}-1}(x+a)^{2 q_{0}}\right.} \\
& \left.+b^{q_{0}}+a^{q_{0}}+b^{q_{0}-1} a^{2 q_{0}}\right],
\end{aligned}
$$

$P_{(a, c)}$ being a zero of $h_{0}$. Since $\mu \notin K$, it follows that $v_{P_{1}}\left(h_{0}\right)=v_{P_{2}}\left(h_{0}\right)=q_{0}$. Now by using the system (5.2) with $\mu^{-1}$ instead of $\mu$ we find the remaining two zeroes of $h_{0}$, namely

$$
P_{3}:=P_{\left(\mu^{-1}, \lambda^{q} \mu^{-1}\right)}, \quad P_{4}:=P_{\left(\mu^{-1}, \lambda^{q} \mu^{-1}+b\right)} ;
$$

moreover, $v_{P_{3}}\left(h_{0}\right)=v_{P_{4}}\left(h_{0}\right)=q_{0}$ and hence

$$
\phi^{*}(X)=\left(q_{0}-1\right) P_{1}+\left(q_{0}-1\right) P_{2}+q_{0} P_{3}+q_{0} P_{4}+\sum_{i=1}^{q-2 q_{0}+1} \mathbf{B}^{i}\left(A_{0}\right) .
$$


Next we compute the intersection divisor of $X$ and $\mathcal{C}$ : we have

$$
X \cdot \mathcal{C}=\sum_{i=1}^{q-2 q+1}\left(0: \lambda^{i}: \lambda^{-i}\right)+\left(2 q_{0}-1\right) Q_{1}+\left(2 q_{0}-1\right) Q_{2},
$$

where $Q_{1}:=\phi\left(P_{1}\right)=\phi\left(P_{4}\right)=(0: 1: 0)$ and $Q_{2}:=\phi\left(P_{2}\right)=\phi\left(P_{3}\right)=(0: 0: 1)$ (from (4.2) and the fact that $h_{1}=\lambda^{2} h_{2}+\left(\lambda^{2}+1\right)(x+\mu)$ ). Then we may assume

$$
f_{1}(Y, Z)=f_{2}(Y, Z)+\left(f_{3}(Y, Z)+(Y Z)^{2 q_{0}-1}\right)\left(Y^{q-2 q_{0}+1}+Z^{q-2 q_{0}+1}\right),
$$

where $\operatorname{deg}\left(f_{2}(Y, Z)\right)<q+2 q_{0}-1$ and $\operatorname{deg}\left(f_{3}(Y, Z)\right)<4 q_{0}-2$. Now by using the action of the automorphisms on $\mathcal{C}$ induced by $\mathbf{B}$ and $\mathbf{W} \circ \mathbf{B} \in \operatorname{Aut}(\mathcal{S})$ via $(X: Y: Y) \mapsto\left(X: \lambda Y: \lambda^{-1} Z\right)$ and $(X: Y: Z) \mapsto(X: Z: Y)$ respectively, we can further assume that $f_{2}(Y, Z)=\widetilde{f}_{2}(Y Z)$ and $f_{3}(Y, Z)=\widetilde{f}_{3}(Y Z)$ with $\widetilde{f}_{2}(t), \widetilde{f}_{3}(t) \in \mathbb{F}_{q^{4}}[t]$.

Claim 5.6.

(1) With $f(t)$ defined in (4.1), there exists e $\in \mathbb{F}_{q^{4}}^{*}$ such that ef $(t)=\widetilde{f}_{2}(t)$.

(2) There exists $e^{\prime} \in \mathbb{F}_{q^{4}}$ such that $\widetilde{f}_{3}(t)+t^{2 q_{0}-1}=e^{\prime}\left(t^{q_{0}}+t^{2 q_{0}-1}\right)$.

Proof. (1) The proof is similar to that of Claims 4.4 and 5.4.

(2) Let $\xi:=h_{1} / h_{0}$ and $\zeta:=h_{2} / h_{0}$ be the rational functions on $\mathcal{S}$ defining the function field of $\mathcal{S}$. Thus

$$
e f(\xi \zeta)=\left(\widetilde{f}_{3}(\xi \zeta)+(\xi \zeta)^{2 q_{0}-1}\right)\left(\xi^{q-2 q_{0}+1}+\zeta^{q-2 q_{0}+1}\right) .
$$

Let $P_{1}, P_{2}, P_{3}$ and $P_{4}$ be the points in the support of $\phi^{*}(X)$ (see (5.6)). Next we use computations by using the valuation at $P_{1}$ to show first that the order of $\widetilde{f}_{3}(t)$ is $q_{0}-1$. By $(4.2)$ we have

$$
v_{P_{1}}\left(f_{3}(\xi \zeta)+(\xi \zeta)^{2 q_{0}-1}\right)=\left(q_{0}-1\right)\left(q-2 q_{0}+1\right),
$$

and $v_{P_{1}}(\xi \zeta)=q-2 q_{0}+1$; thus the assertion on the order of $\widetilde{f}_{3}(t)$ follows. Now for $a \in \bar{K}$ a root of $\widetilde{f}_{3}(t)+t^{2 q_{0}-1}$, let $\mathcal{C}_{a}$ be the conic defined by the equation $Y Z=a$. We have $\mathcal{C}_{a} \cap \mathcal{C} \subseteq X$; otherwise, $f(a)=0$ by (1), so that $\mathcal{C}_{a}$ would be a component of $\mathcal{C}$, which is a contradiction. Let $\ell$ be a local parameter at $P=P_{(a, b c)}$; then

$$
\begin{aligned}
& h_{0}=\left(b^{q_{0}-1}\left(1+a^{2 q_{0}}\right)+a^{q_{0}}\right) \ell+\cdots, \\
& h_{1}=h_{1}\left(P_{i}\right)+\left(1+\lambda\left(a^{2 q_{0}}+\mu a^{2 q_{0}+1}+\mu b c\right)\right) \ell+\cdots, \\
& h_{2}=h_{2}\left(P_{i}\right)+\left(1+\lambda^{-1}\left(a^{2 q_{0}}+\mu a^{2 q_{0}+1}+\mu b c\right)\right) \ell+\cdots,
\end{aligned}
$$

and it follows that $v_{P}(\xi \zeta-P)>0$ for some $i$ and also that $a=0$ or 1 . Thus the proof of Claim 5.6 is complete. 
We have shown so far that $\mathcal{C}$ can be defined over $\mathbb{F}_{q^{4}}$ by a polynomial of type

$$
f_{1}(Y, Z)=c f(Y Z)+\left((Y Z)^{q_{0}-1}+(Y Z)^{2 q_{0}-1}\right)\left(Y^{q-2 q_{0}+1}+Z^{q-2 q_{0}+1}\right) .
$$

Finally, we prove that $c=b$; we use local computations at $P_{(0,0)} \in \mathcal{S}$ via the rational functions $\xi=h_{1} / h_{0}$ and $\zeta=h_{2} / h_{0}$ (recall that $x$ is a local parameter at that point). By the definition of $h_{0}, h_{1}$ and $h_{2}$,

$$
\begin{aligned}
& h_{0}=b^{q_{0}-1} x+x^{q_{0}+1}+\cdots, \\
& h_{1}=\mu+x+\lambda x^{2 q_{0}+1}+\cdots, \\
& h_{2}=\mu+x+\lambda^{-1} x^{2 q_{0}+1}+\cdots,
\end{aligned}
$$

whence

$$
\xi=\left(\mu / b^{q_{0}-1}\right) x^{-1}(1+\cdots), \quad \zeta=\left(\mu / b^{q_{0}-1}\right) x^{-1}(1+\cdots) .
$$

Now by means of (5.7) we compare the order at 0 of the local power series $c \varrho^{2} \xi \zeta f\left(\varrho^{2} \xi \zeta\right)$ and $\left(\left(\varrho^{2} \xi \zeta\right)^{q_{0}}+\left(\varrho^{2} \xi \zeta\right)^{2 q_{0}}\right)\left(\xi^{q-2 q_{0}+1}+\zeta^{q-2 q_{0}+1}\right) \varrho^{q-2 q_{0}+1}$, where $\varrho:=b^{-q_{0}} \mu^{-1}$. The order at 0 of the former series is $c / b^{2 q_{0}-1}$; for the latter, it is enough to compute the order at 0 of

$$
\left(\varrho^{2} \xi \zeta\right)^{q_{0}}\left(\xi^{q-2 q_{0}+1}+\zeta^{q-2 q_{0}+1}\right) \varrho^{q-2 q_{0}+1} .
$$

By (5.8) and some computations,

$$
\frac{h_{1}^{q-2 q_{0}+1}+h_{2}^{q-2 q_{0}+1}}{h_{0}^{q-2 q_{0}+1}}=\frac{1}{b^{6\left(q_{0}-1\right)} \varrho} x^{-q+4 q_{0}}+\cdots ;
$$

thus we obtain

$$
\frac{c}{b^{2 q_{0}-1}}=\left(\frac{1}{b^{4 q_{0}-2}}\right)^{2 q_{0}} b^{-6\left(q_{0}-1\right)}
$$

so that $c=b$. Hence the following holds.

Lemma 5.7. The Suzuki curve $\mathcal{S}$ admits a plane model over $\mathbb{F}_{q^{4}}$ defined by

$$
f_{1}(Y, Z)=b f(Y Z)+\left((Y Z)^{q_{0}-1}+(Y Z)^{2 q_{0}-1}\right)\left(Y^{q-2 q_{0}+1}+Z^{q-2 q_{0}+1}\right),
$$

where $f(t)$ is as in (4.1), and $b=\lambda^{q_{0}}+\lambda^{q_{0}-1}+\lambda^{-q_{0}}+\lambda^{-\left(q_{0}-1\right)}$ with $\lambda \in \mathbb{F}_{q^{4}}$ of order $q-2 q_{0}+1$.

Proof of Theorem 5.1. The genus $\widetilde{g}$ : By the proof of Claim 5.2 (see (5.3) and (5.6) above), the natural morphism $\mathcal{S} \rightarrow \widetilde{\mathcal{S}}$ is unramified or totally ramified precisely at four points according as $\mathcal{U} \subseteq \mathcal{S}_{1}$ or $\mathcal{U} \subseteq \mathcal{S}_{-1}$. Then the formula for $\widetilde{g}$ is computed by the Riemann-Hurwitz genus formula.

The plane equation: Let $\widetilde{\mathcal{C}}$ be the absolutely irreducible plane curve over $K$ whose function field is $K\left(Y Z, Z^{r}\right)$. By the definition of $\mathcal{S}_{ \pm 1}$, we have the 
inclusion $K\left(Y Z, Z^{r}\right) \subseteq K(Y, Z)^{\mathcal{U}}$. On the other hand, from the definition of $f_{1}(X, Z)$ in Lemmas 5.5 and 5.7 ,

$$
\left[K(Y, Z): K\left(Y Z, Z^{r}\right)\right]=\left[K(Y Z, Z): K\left(Y Z, Z^{r}\right)\right]=r
$$

and thus the inclusion is an equality. Taking $U:=Y Z$ and $V:=Z^{r}$ we obtain the required equation for $\widetilde{\mathcal{C}}$, which is clearly a plane model of $\widetilde{\mathcal{S}}$.

Remark 5.8. Let $P_{1}, P_{2}, P_{3}$ and $P_{4}$ be the points defined in (5.6), and $L$ the line through the points $P_{1}$ and $P_{2}$. This line is fixed by the automorphism $\mathbf{B}$ defined above, and $\phi$ is the morphism defined by the linear series cut out on $\mathcal{S}$ by hyperplanes through $L$. By the proof of Claim 5.2, (5.3) and (5.6), the natural morphism $\mathcal{S} \rightarrow \widetilde{\mathcal{S}}$ is unramified or totally ramified at the aforementioned points according as $\mathcal{U} \subseteq \mathcal{S}_{1}$ or $\mathcal{U} \subseteq \mathcal{S}_{-1}$.

REMARK 5.9. The curve in Theorem 5.1 covers a hyperelliptic curve of genus $q_{0}$ defined by $v^{2}+v=u^{q+2 q_{0}+1} / \widetilde{f}(u)^{2}$ in case $(1)$ and by $v^{2}+b v=$ $\left(u^{q_{0}-1}+u^{2 q_{0}-1}\right)^{2} u^{q-2 q_{0}+1} / f(u)^{2}$ in case $(2)$.

6. Non-tame quotient curves of the Suzuki curve. In this section we investigate quotient curves of the Suzuki curve $\mathcal{S}$ arising from the non-tame subgroups of $\operatorname{Aut}(\mathcal{S})$. Non-tame ramifications together with a huge number of pairwise non-conjugate subgroups of even order (especially 2-subgroups) do not allow us to obtain results as complete as those achieved in Sections 4 and 5. Nevertheless, we manage to compute the degree of the ramification divisor of the relevant natural morphism and thus the genus of such curves; in several cases we will also provide plane models.

From Section 2, the subgroups of $\mathcal{S} z(q)$ of even order are of the following types, up to conjugacy in $\mathcal{S} z(q)$ :

I. Subgroups of $\widetilde{\mathbf{T}}$;

II. Subgroups of order $2^{v} r$ with $v>1$ and $r>1$ a divisor of $q-1$;

III. Dihedral subgroups of order $2 r$ with $r>1$ a divisor of $q-1$;

IV. Subgroups of order $2 r$ with $r>1$ a divisor of $q \pm 2 q_{0}+1$;

V. Subgroups of order $4 r$ with $r>1$ a divisor of $q \pm 2 q_{0}+1$;

VI. Subgroups isomorphic to $\mathcal{S} z(\bar{q})$.

Type $I$. Let $\mathcal{U}$ be a subgroup of $\widetilde{\mathbf{T}}$. We shall compute the genus $\widetilde{g}$ of the quotient curve $\widetilde{\mathcal{S}}=\mathcal{S} / \mathcal{U}$, by considering the subgroup $\mathcal{U}_{2}$ of $\mathcal{U}$ consisting of the elements of even order together with the identity (a similar problem on the Hermitian curve was considered in [7]). Let $u$ and $v$ be the integers such that $2^{u}:=\# \mathcal{U}_{2}$ and $2^{v}:=\# \mathcal{U}$. Recall that $q=2 q_{0}^{2}$ and $q_{0}=2^{s}, s \in \mathbb{N}$. 
TheOREM 6.1. With the notation above,

$$
\widetilde{g}=\frac{q_{0}}{2^{v-u}}\left(\frac{q}{2^{u}}-1\right) ;
$$

in particular, $u \leq 2 s+1$ and $v-u \leq s$.

Proof. The Riemann-Hurwitz formula asserts that

$$
2 q_{0}(q-1)-2=2^{v}(2 \widetilde{g}-2)+\operatorname{deg}(R),
$$

where $R$ is the associated ramification divisor of the natural morphism $\mathcal{S} \rightarrow \widetilde{\mathcal{S}}$. Let $\ell$ be a local parameter at $P \in \mathcal{S}$; we have $v_{P}(R)=\sum_{\mathbf{T}} i_{\mathbf{T}, P}$, where the summation is extended over all $\mathbf{T} \in \mathcal{U}$ such that $\mathbf{T}(P)=P$, and where $i_{\mathbf{T}, P}=v_{P}\left(\mathbf{T}^{*}(\ell)-\ell\right)$ (see e.g. [31, III.8.8]). By the definition of $\mathbf{T}=\mathbf{T}_{a, c}$ (cf. Section 2), $v_{P}(R)=0$ for $P \neq A_{4}$. On the other hand, at the point $A_{4}$ the rational function $z / w$ is a local parameter by (4.2); therefore

$$
i_{\mathbf{T}, A_{4}}= \begin{cases}2 q_{0}+2 & \text { for } a=0, \\ 2 & \text { for } a \neq 0 .\end{cases}
$$

We see that $\mathbf{T} \neq 1$ is an element of order 2 for $a=0$, otherwise its order is 4 . Thus the Riemann-Hurwitz formula above becomes

$$
2 q_{0}(q-1)-2=2^{v}(2 \widetilde{g}-2)+\left(2 q_{0}+2\right)\left(2^{u}-1\right)+2\left(2^{v}-2^{u}\right),
$$

and we obtain the formula for $\widetilde{g}$. The numerical conditions follow from the fact that $\widetilde{g}$ must be a non-negative integer.

In Corollary 6.5 we are going to point out sufficient conditions for the existence of non-tame quotient curves of $\mathcal{S}$ whose genus can be computed via Theorem 6.1 . For some cases regarding $v=u$ and $(v, u)=(2,1)$, we can exhibit a plane model; see Theorem 6.6.

Theorem 6.1 raises the problem of classifying the subgroups of $\widetilde{\mathbf{T}}$ in terms of their elements of order 2 . Such a general problem is computationally beyond our reach, because $\widetilde{\mathbf{T}}$ contains a huge number of pairwise non-conjugate subgroups. The following lemma states some (necessary) numerical conditions on $u$ and $v$.

Lemma 6.2. Let $\mathcal{U}$ be a subgroup of $\widetilde{\mathbf{T}}$ and $\mathcal{U}_{2}$ the subgroup of its elements of even order. Let $2^{v}$ and $2^{u}$ be the orders of $\mathcal{U}$ and $\mathcal{U}_{2}$ respectively. Then:

(1) $v \leq 2 u$.

(2) For every integer $u^{\prime}$ with $u \leq u^{\prime} \leq v$ there is a subgroup of $\mathcal{U}$ of order $2^{u^{\prime}}$ containing $\mathcal{U}_{2}$. In particular, for each integer $u^{\prime}$ with $2 s+1 \leq u^{\prime} \leq 4 s+2$, there is a subgroup of $\widetilde{\mathbf{T}}$ of order $2^{u^{\prime}}$ containing all elements of $\widetilde{\mathbf{T}}$ of order 2 . 
Proof. (1) We consider the homomorphism of groups

$$
\Phi=\Phi_{\mathcal{U}}: \mathcal{U} \rightarrow K, \quad T_{a, c} \mapsto a,
$$

where $K$ is equipped with its additive structure. We have $\operatorname{Ker}(\Phi)=\mathcal{U}_{2}$ and thus $\mathcal{U} / \mathcal{U}_{2}$ is isomorphic to $\Phi(\mathcal{U})$. As the map $K \rightarrow K, a \mapsto a^{2 q_{0}+1}$, is injective, and as $\mathbf{T}_{a, c}^{2}=\mathbf{T}_{0, a^{2 q_{0}+1}}$, we have $\# \Phi(\mathcal{U}) \leq \# \mathcal{U}_{2}$ and hence the assertion.

(2) Since the quotient $\mathcal{U} / \mathcal{U}_{2}$ is an elementary abelian group, the converse of the Lagrange theorem holds and the first statement follows; for the second statement take $\mathcal{U}=\widetilde{\mathbf{T}}$; cf. (2.3).

Sufficient conditions for the existence of a subgroup $\mathcal{U}$ of $\widetilde{\mathbf{T}}$ with a given subgroup $\mathcal{U}_{2}$ is ensured by the following lemma. Let $\Phi_{\mathcal{U}}$ be the map defined in the proof of Lemma 6.2.

LeMma 6.3.

(1) Let $\mathcal{V}$ be an elementary abelian group of $\widetilde{\mathbf{T}}$ of order $2^{u}$. Then there exists a subgroup $\mathcal{U}$ of $\widetilde{\mathbf{T}}$ of order $2^{u+1}$ such that $\mathcal{U}_{2}$ coincides with $\mathcal{V}$.

(2) Let $v \geq u \geq 0$ be integers, and $\mathcal{B}$ an additive subgroup of $K$ of order $2^{u}$. If there exists an additive subgroup $\mathcal{A}$ of $K$ of order $2^{v-u}$ such that $\mathcal{A}^{2 q_{0}+1} \subseteq \mathcal{B}$, then there exists a subgroup $\mathcal{U}$ of $\widetilde{\mathbf{T}}$ of order $2^{v}$ such that $\Phi_{\mathcal{U}}(\mathcal{U})=\mathcal{A}$ and $\operatorname{Ker}\left(\Phi_{\mathcal{U}}\right)=\left\{\mathbf{T}_{0, c}: c \in \mathcal{B}\right\}$ (in particular, $\left.\operatorname{ord}\left(\mathcal{U}_{2}\right)=2^{u}\right)$.

Proof. (1) Since the normalizer in $\operatorname{Aut}(\mathcal{S})$ of $\widetilde{\mathbf{T}}$ acts transitively on the set of elements of order 2 of $\widetilde{\mathbf{T}}$ (cf. (2.3)) we may assume $\mathbf{T}_{0,1} \notin \mathcal{V}$. Then the group $\mathcal{U}$ generated by $\mathcal{V}$ and $\mathbf{T}_{0,1}$ fulfills the required conditions.

(2) The proof of this assertion is by induction on $v \geq u$. If $v=u$, then $\mathcal{A}=\{0\}$ and $\mathcal{U}:=\left\{\mathbf{T}_{0, c}: c \in \mathcal{B}\right\}$ have the required properties. Suppose now that $v>u$. As $\mathcal{A}$ is an elementary abelian group, it contains a subgroup $\mathcal{A}_{0}$ of index 2, that is, of order $2^{(v-1)-u}$. Since $\mathcal{A}_{0}^{2 q_{0}+1} \subseteq \mathcal{A}^{2 q_{0}+1}$, there is by induction a subgroup $\mathcal{U}_{0}$ in $\widetilde{\mathbf{T}}$ of order $2^{v-1}$ with $\Phi_{\mathcal{U}_{0}}\left(\mathcal{U}_{0}\right)=\mathcal{A}_{0}(*)$ and $\operatorname{Ker}\left(\Phi_{\mathcal{U}_{0}}\right)=\left\{\mathbf{T}_{0, c}: c \in \mathcal{B}\right\}(* *)$. Fix $\mathbf{T}:=\mathbf{T}_{a, c} \in \widetilde{\mathbf{T}}$ with $a \in \mathcal{A} \backslash \mathcal{A}_{0}$ which does not belong to $\mathcal{U}_{0}$ by $(*)$. Let $\mathcal{U}$ be the subgroup of $\widetilde{\mathbf{T}}$ generated by $\mathcal{U}_{0}$ together with $\mathbf{T}$.

To prove that the order of $\mathcal{U}$ is $2^{v}$ we show that $\mathbf{T} \mathcal{U}_{0}=\mathcal{U}_{0} \mathbf{T}$. Let $\mathbf{T}_{0}:=$ $\mathbf{T}_{a_{0}, c_{0}}$ be any element of $\mathcal{U}_{0}$. Note that $\left(\mathbf{T} \circ \mathbf{T}_{0}\right)^{2}=\mathbf{T}_{0,\left(a+a_{0}\right)^{2 q_{0}+1}} \in \mathcal{U}_{0}$. In fact, $\left(a+a_{0}\right)^{2 q_{0}+1} \in \mathcal{B}$ since $a+a_{0} \in \mathcal{A}$, and the claim follows by (**). Now, as $\widetilde{\mathbf{T}}$ has exponent 4 and as every element of order two in $\widetilde{\mathbf{T}}$ is in the 
center $Z(\widetilde{\mathbf{T}})($ cf. $(2.3))$ we have

$\mathbf{T} \circ \mathbf{T}_{0}=\mathbf{T} \circ \mathbf{T}_{0}\left(\mathbf{T} \circ \mathbf{T}_{0}^{4} \circ \mathbf{T}^{3}\right)=\left(\mathbf{T} \circ \mathbf{T}_{0}\right)^{2} \mathbf{T}_{0}^{3} \circ \mathbf{T}^{3}=\left(\mathbf{T} \circ \mathbf{T}_{0}\right)^{4} \circ \mathbf{T}_{0} \circ \mathbf{T} \in \mathcal{U}_{0} \mathbf{T}$.

Finally, $\Phi_{\mathcal{U}}\left(\mathbf{T} \circ \mathbf{T}_{0}\right)=\Phi_{\mathcal{U}}(\mathbf{T})+\Phi_{\mathcal{U}}\left(\mathbf{T}_{0}\right)=a+a_{0}$ implies $\Phi_{\mathcal{U}}(\mathcal{U})=\mathcal{A}$ and $\operatorname{Ker}\left(\Phi_{\mathcal{U}}\right) \subseteq \operatorname{Ker}\left(\Phi_{\mathcal{U}_{0}}\right)$, and the result follows.

REMARK 6.4. Lemma 6.3(1) does not hold true for subgroups $\mathcal{U}$ of order $2^{u+\ell}$ with $\ell>1$, as the following example shows. Fix an element $e \in K \backslash \mathbb{F}_{2}$. The set $\mathcal{V}=\left\{\mathbf{T}_{0,0}, \mathbf{T}_{0,1}, \mathbf{T}_{0, e}, \mathbf{T}_{0, e+1}\right\}$ is an elementary abelian subgroup of $\widetilde{\mathbf{T}}$ of order $2^{v}$ with $v=2$. Assume that there is a subgroup $\mathcal{U}$ of $\widetilde{\mathbf{T}}$ of order $2^{4}$ such that $\mathcal{U}_{2}=\mathcal{V}$. Then there are three pairwise distinct non-zero elements $a_{1}, a_{2}, a_{3} \in K$ and three elements $c_{1}, c_{2}, c_{3} \in K$ such that $\mathbf{T}_{a_{i}, c_{i}}(i=1,2,3)$ together with $\mathbf{T}_{0,0}$ form a complete set of representatives of the cosets of $\mathcal{U} / \mathcal{V}$. Furthermore, $\Phi_{\mathcal{U}}(\mathcal{U})=\left\{0, a_{1}, a_{2}, a_{3}\right\}$ and $a_{3}=a_{1}+a_{2}(*)$. On the other hand, we can assume $a_{1}=1, a_{2}^{2 q_{0}+1}=e$, $a_{3}^{2 q_{0}+1}=e+1$ since $\mathbf{T}_{a_{i}, c_{i}}^{2}=T_{0, a_{i}^{2 q_{0}+1}}$. Then $(*)$ implies $a_{3}^{2 q_{0}}+a_{3}=0$, a contradiction.

Corollary 6.5. Let $v \geq u \geq 0$ be integers such that $v-u \leq s, u \leq 2 s+1$ and $v \leq 2 u$. Then there exists a non-tame quotient curve of $\mathcal{S}$ whose genus is given by Theorem 6.1 provided that

$$
v-u \leq \log _{2}(u+1) \quad \text { or } \quad(v-u) \mid(2 s+1) .
$$

Proof. Suppose at first that both $v-u \leq 2 s+1$ and $v-u \leq \log _{2}(u+1)$ hold. For any additive subgroup $\mathcal{A}$ of $K$ of order $2^{v-u}$, the additive subgroup $\mathcal{B}^{\prime}$ of $K$ generated by all elements in $\mathcal{A}^{2 q_{0}+1}$ has order at most $2^{2^{v-u}-1}$. In fact, $K$ can be viewed as a vector space over its subfield $\mathbb{F}_{2}$, and the subspace generated by $\mathcal{A}^{2 q_{0}+1}$ has dimension at most $2^{v-u}-1$. Then there exists an additive subgroup $\mathcal{B}$ of $K$ of order $2^{u}$ containing $\mathcal{B}^{\prime}$, and the claim follows from Lemma 6.3(2).

Now suppose that $(v-u) \mid(2 s+1)$ and $v \leq 2 u$. Then $\mathbb{F}_{2^{v-u}}$ is a subfield of $K$. Let $\mathcal{B}$ be any additive subgroup of order $2^{u}$ containing the additive group $\mathcal{A}$ of $\mathbb{F}_{2^{v-u}}$. Again Lemma 6.3(2) proves the assertion.

In some cases we are also able to provide a plane model for $\widetilde{\mathcal{S}}=\mathcal{S} / \mathcal{U}$.

THEOREM 6.6. With the notation above:

(1) Let $q=\widetilde{q}^{n}$ and $\mathcal{U}$ be the elementary abelian subgroup of $\widetilde{\mathbf{T}}$ consisting of all automorphisms $\mathbf{T}_{0, c}$ with $c \in \mathbb{F} \widetilde{q}$. Then the curve $\widetilde{\mathcal{S}}$ has genus $\widetilde{g}=q_{0}(q / \widetilde{q}-1)$, and a plane model over $K$ of $\widetilde{\mathcal{S}}$ is given by

$$
\sum_{i=0}^{n-1} V^{\widetilde{q}^{i}}=U^{2 q_{0}}\left(U^{q}+U\right)
$$


(2) For a cyclic subgroup $\mathcal{U}$ of $\operatorname{Aut}(\mathcal{S})$ of order 4 , the curve $\widetilde{\mathcal{S}}$ has genus $\widetilde{g}=\frac{1}{4} q_{0}(q-2)$, and a plane model over $K$ of $\widetilde{\mathcal{S}}$ is given by

$$
\sum_{i=0}^{2 s} V^{2^{i}}=\sum_{i=0}^{2 s} U^{2^{i}}+\sum_{i=0}^{s}\left(\sum_{j=i}^{s} U^{2^{j}}\right) U^{2^{i}}+\sum_{i=s+2}^{2 s}\left(\sum_{j=0}^{i-s-2} U^{2^{j}}\right)^{2 q_{0}} U^{2^{i}} .
$$

Proof. (1) We have $\mathcal{U}=\mathcal{U}_{2}$ and the formula for the genus follows from Theorem 6.1. We now consider the morphism $\phi:=\left(1: x: z^{\widetilde{q}}+z\right): \mathcal{S} \rightarrow \mathbb{P}^{2}$; then $\widetilde{\mathcal{S}}$ is the non-singular model over $K$ of $\phi(\mathcal{S})$ since $\phi^{-1}\left(\phi\left(P_{(a, c)}\right)\right)=$ $\left\{P_{(a, c+e)}: e \in \mathbb{F}_{\widetilde{q}}\right\}$. To write a plane equation for $\phi(\mathcal{S})$ we use $(3.5)$ with $U:=x$ and $V:=z^{\widetilde{q}}+z$ taking into account that $z^{q}+z=\left(z^{\widetilde{q}}+z\right)+$ $\left(z^{\widetilde{q}}+z\right)^{\widetilde{q}}+\cdots+\left(z^{\widetilde{q}}+z\right)^{\widetilde{q}^{n-1}}$.

(2) Since the cyclic subgroups of $\operatorname{Aut}(\mathcal{S})$ of order 4 are pairwise conjugate in $\operatorname{Aut}(\mathcal{S})($ cf. $(2.4))$, we may assume $\mathcal{U}$ to be generated by $\mathbf{T}_{1,0}$. Here $\mathcal{U}_{2}$ has two elements and the formula for $\widetilde{g}$ follows from Theorem 6.1. We now consider the morphism $\phi:=\left(1: x^{2}+x: x^{3}+x+z^{2}+z\right): \mathcal{S} \rightarrow \mathbb{P}^{2}$. Then $\widetilde{\mathcal{S}}$ is the non-singular model over $K$ of $\phi(\mathcal{S})$ since $\phi^{-1}\left(\phi\left(P_{(a, c)}\right)\right)=$ $\left\{\mathbf{T}_{1,0}^{i}: i=1, \ldots, 4\right\}$. To write a plane equation for $\phi(\mathcal{S})$ we notice that

$$
\sum_{i=0}^{2 s}\left(x^{3}+x+z^{2}+z\right)^{2^{i}}=\sum_{i=0}^{2 s}\left(x^{3}+x\right)^{2^{i}}+z^{q}+z ;
$$

therefore, with $U:=x^{2}+x$ and $V:=x^{3}+x+z^{2}+z$, by the equalities (3.5), $x^{q}+x=\sum_{i=0}^{2 s}\left(x^{2}+x\right)^{2^{i}}$ and $x^{3}+x=\left(x^{2}+x\right)+\left(x^{3}+x^{2}\right)$,

$$
\sum_{i=0}^{2 s} V^{2^{i}}=\sum_{i=0}^{2 s} U^{2^{i}}+\sum_{i=0}^{2 s}\left(x^{2^{i}}+x^{2 q_{0}}\right) U^{2^{i}}
$$

Now the claimed equation follows from the relations

$$
x^{2^{i}}+x^{2 q_{0}}= \begin{cases}\sum_{j=i}^{s}\left(x^{2}+x\right)^{2^{j}} & \text { if } i<s+1, \\ 0 & \text { if } i=s+1, \\ \left(\sum_{j=0}^{i-s-2}\left(x^{2}+x\right)^{2^{j}}\right)^{2 q_{0}} & \text { if } i>s+1 .\end{cases}
$$

Type II. The basic fact here is that, up to conjugacy in $\operatorname{Aut}(\mathcal{S})$, a subgroup $\mathcal{U}$ of $\operatorname{Aut}(\mathcal{S})$ of order $2^{v} r(v, r>1, r \mid(q-1))$ is contained in $\widetilde{\mathbf{T}} \widetilde{\mathbf{N}}$; moreover, the orders of its elements are known (cf. (2.5)).

TheOREM 6.7. For a subgroup $\mathcal{U}$ of $\widetilde{\mathbf{T}} \widetilde{\mathbf{N}}$ of order $2^{v} r$ with $v, r>1$ and $r \mid(q-1)$, let the subgroup $\mathcal{U}_{2}$ of $\mathcal{U}$ consist of all elements of order 2 . If $\mathcal{U}_{2}$ has order $2^{u}$, then

$$
\widetilde{g}=\frac{q_{0}}{2^{v-u r}}\left(\frac{q}{2^{u}}-1\right) ;
$$

in particular, $u \leq 2 s+1, v-u \leq s$ and $r$ divides $q / 2^{u}-1$. 
Proof. The Riemann-Hurwitz genus formula states

$$
2 q_{0}(q-1)-2=2^{v} r(2 \widetilde{g}-2)+\operatorname{deg}(R),
$$

where $R$ is the ramification divisor of the natural morphism $\mathcal{S} \rightarrow \widetilde{\mathcal{S}}$. We have to look at the points $P \in \mathcal{S}$ for which there exists $\mathbf{T} \in \mathcal{U}$ with $\mathbf{T}(P)=P$, and compute $i_{\mathbf{T}, P}=v_{P}\left(\mathbf{T}^{*}(\ell)-\ell\right), \ell$ being a local parameter at $P$; by $(4.2)$ the only possible fixed point of $\mathcal{U}$ is $A_{4}$. By the computation in the proof of Theorem 6.1,

$$
\operatorname{deg}(R)=\left(2 q_{0}+2\right)\left(2^{u}-1\right)+2\left(2^{v}-2^{u}\right)+\sum i_{\mathbf{T}, A_{4}},
$$

where the summation is extended over all the automorphisms $\mathbf{T} \in \mathcal{U} \backslash \widetilde{\mathbf{T}}$ such that $\mathbf{T}\left(A_{4}\right)=A_{4}$; from (4.2) we obtain $i_{\mathbf{T}, A_{4}}=2$ and the formula for $\widetilde{g}$ follows. The numerical conditions follow from the fact that $\widetilde{g}$ is a non-negative integer.

Corollary 6.8. Let $u, v, r>1$ be integers such that $v \geq u \geq 0$, $u \leq 2 s+1, v-u \leq s$ and $r$ is a divisor of $q-1$ and $q / 2^{u}-1$. Then there is a non-tame quotient curve of $\mathcal{S}$ whose genus is given by Theorem 6.7 provided that (6.1) holds true.

Proof. This follows from $6.3(2)$ and the structure of $\widetilde{\mathbf{T}} \widetilde{\mathbf{N}}$.

Type III. The basic fact here is that each subgroup of $\operatorname{Aut}(\mathcal{S})$ of order $2 r$, $r>1$ and $r \mid(q-1)$, is conjugate to a subgroup of the dihedral subgroup $N_{\text {Aut }(\mathcal{S})}(\mathbf{N})$; cf. (2.6). We show the following.

THEOREM 6.9. Let $\mathcal{U}$ be a subgroup of $\operatorname{Aut}(\mathcal{S})$ of order $2 r$ with $r>1$ a divisor of $q-1$. Then the quotient curve $\widetilde{\mathcal{S}}=\mathcal{S} / \mathcal{U}$ has genus

$$
\widetilde{g}=\frac{q_{0}}{2}\left(\frac{q-1}{r}-1\right),
$$

and a plane model over $K$ of $\widetilde{\mathcal{S}}$ is given by

$$
f(V)=\sum(-1)^{i+j} \frac{(i+j-1) !}{i ! j !} U^{i} V^{r j}\left(1+V^{q_{0}}\right),
$$

where the summation is extended over all pairs $(i, j)$ of non-negative integers with $i+2 j=(q-1) / r$, and $f(t)$ is the polynomial defined in (4.1).

Proof. The genus: The subgroup $N_{\operatorname{Aut}(\mathcal{S})}(\tilde{\mathbf{N}})$ is a dihedral group of order $2(q-1)$ which comprises $\widetilde{\mathbf{N}}$ together with a coset consisting entirely of elements of order 2 . Hence $\mathcal{U}$ has $r-1$ non-trivial elements of odd order and each of the remaining $r$ elements in $\mathcal{U}$ has order 2. Thus, by (4.2), the degree of the ramification divisor of the natural morphism $\mathcal{S} \rightarrow \widetilde{\mathcal{S}}$ is $\left(2 q_{0}+2\right) r+2(r-1)$ and the result follows from the Riemann-Hurwitz genus formula. 
A plane model: Let $\psi:=\widetilde{\phi} \circ \phi: \mathcal{S} \rightarrow \mathbb{P}^{2}$, where $\phi$ is the morphism defined in Section 4 and $\widetilde{\phi}$ is the morphism on $\phi(\mathcal{S})$ defined by $(X: 1: Z) \mapsto$ $\left(1: X Z: X^{r}+Z^{r}\right)$. We claim that $\widetilde{\mathcal{S}}$ is the non-singular model over $K$ of $\psi(\mathcal{S})$. Since $\phi$ is birational (see Lemma 4.5 ), to prove this claim it is enough to check that $\# \widetilde{\phi}^{-1}(\widetilde{\phi}(P))=2 r$ for a (generic) point $P=(a: 1: c)$ $\in \phi(\mathcal{C})$. We have $\left(a \tau^{-i}: 1: c \tau^{i}\right),\left(c \tau^{i}: 1: a \tau^{-i}\right) \in \widetilde{\phi}^{-1}(\widetilde{\phi}(P))(i=1, \ldots, r)$, where $\tau$ is an element of order $r$ in $K^{*}$. On the other hand, let $P^{\prime}=$ $(\widetilde{a}: 1: \widetilde{c})$ be such that $\widetilde{\phi}\left(P^{\prime}\right)=\widetilde{\phi}(P)$. Then $\widetilde{a}^{r}$ and $\widetilde{c}^{r}$ are the roots of $W^{2}+\left(a^{r}+c^{r}\right) W+a^{r} c^{r}=0$ and hence the claim is proved.

To find an equation of $\psi(\mathcal{S})$ we start from the equation defining $\phi(\mathcal{S})$ in Lemma 4.5:

$$
f(\widetilde{X} \widetilde{Y})=\left(1+(\widetilde{X} \widetilde{Y})^{q_{0}}\right)\left(\left(\widetilde{X}^{r}\right)^{(q-1) / r}+\left(\widetilde{Y}^{r}\right)^{(q-1) / r}\right) .
$$

Thus we need a formula relating the form $A^{m}+B^{m}$ to polynomials of type $A+B$ and $A^{i} B^{i}$. We can do that by means of Waring's formula in two indeterminates over a finite field [22, Thm. 1.76]:

$$
A^{m}+B^{m}=\sum(-1)^{i+j} \frac{(i+j-1) ! m}{i ! j !}(A+B)^{i}(A B)^{j},
$$

where the summation is extended over all pairs $(i, j)$ of non-negative integers for which $i+2 j=m$. Now the result follows by taking $m=(q-1) / r \equiv 1$ $(\bmod 2), U:=\widetilde{X}^{r}+\widetilde{Y}^{r}$ and $V:=\widetilde{X} \widetilde{Y}$.

Type $I V$. We use the fact that each subgroup of $\operatorname{Aut}(\mathcal{S})$ of order $2 r$ with $r>1$ and $r \mid\left(q \pm 2 q_{0}+1\right)$ is conjugate under $\operatorname{Aut}(\mathcal{S})$ to a subgroup of the dihedral subgroup of $\operatorname{Aut}(\mathcal{S})$ which comprises the Singer subgroups $\mathcal{S}_{ \pm 1}$ together with a coset consisting entirely of non-trivial elements of order 2 ; cf. (2.7).

Theorem 6.10. Let $r>1$ be an integer and $\mathcal{U}$ a subgroup of $\operatorname{Aut}(\mathcal{S})$ of order $2 r$.

(1) If $r$ is a divisor of $q+2 q_{0}+1$, the quotient curve $\widetilde{\mathcal{S}}=\mathcal{S} / \mathcal{U}$ has genus

$$
\widetilde{g}=\frac{q_{0}-1}{2}\left(\frac{q+2 q_{0}+1}{r}-1\right) .
$$

Furthermore, a plane model over $\mathbb{F}_{q^{4}}$ of $\widetilde{\mathcal{S}}$ is given by

$$
\widetilde{f}(V)=\sum(-1)^{i+j} \frac{(i+j-1) !}{i ! j !} U^{i} V^{r j}
$$

where the summation is extended over all pairs $(i, j)$ of non-negative integers with $i+2 j=\left(q+2 q_{0}+1\right) / r$, and $\widetilde{f}(t)$ is the polynomial defined in (5.1). 
(2) If $r$ is a divisor of $q-2 q_{0}+1$, the quotient curve $\widetilde{\mathcal{S}}=\mathcal{S} / \mathcal{U}$ has genus

$$
\widetilde{g}=\frac{q_{0}+1}{2}\left(\frac{q-2 q_{0}+1}{r}-1\right) .
$$

Furthermore, a plane model over $\mathbb{F}_{q^{4}}$ of $\widetilde{\mathcal{S}}$ is given by

$$
b f(V)=\sum(-1)^{i+j} \frac{(i+j-1) !}{i ! j !} U^{i} V^{r j}\left(V^{q_{0}-1}+V^{2 q_{0}-1}\right),
$$

where the summation is extended over all pairs $(i, j)$ of non-negative integers with $i+2 j=\left(q-2 q_{0}+1\right) / r, f(t)$ defined in (4.1), and $b$ as in Theorem 5.1(2).

Proof. The genus: The subgroup $\mathcal{U}$ has $r-1$ non-trivial elements of odd order and $r$ of even order; thus the degree of the ramification divisor is $\left(2 q_{0}+2\right) r+\widetilde{R}$ (cf. proof of Theorem 6.1). By (4.2), in case (1) we obtain $\widetilde{R}=0$ and in case $(2), \widetilde{R}=4(r-1)$. Now the result follows from the Riemann-Hurwitz genus formula.

A plane model: To find the equations stated above we argue as in the proof of Theorem 6.9. For case (1), the corresponding morphism $\phi$ is taken to be the one defined in Section 5; we use the plane model of $\mathcal{S}$ in Lemma 5.5 written as

$$
\widetilde{f}(\tilde{X} \tilde{Y})=\left(\widetilde{X}^{r}\right)^{\left(q+2 q_{0}+1\right) / r}+\left(\widetilde{Y}^{r}\right)^{\left(q+2 q_{0}+1\right) / r}
$$

Then we get the claimed equation from (6.2) with $m=\left(q+2 q_{0}+1\right) / r \equiv 1$ $(\bmod 2), U:=\widetilde{X}^{r}+\widetilde{Y}^{r}$ and $V:=\widetilde{X} \widetilde{Y}$. For the case $(2)$ we use the plane model of $\mathcal{S}$ stated in Lemma 5.7.

Type $V$. We use the fact that any subgroup of $\operatorname{Aut}(\mathcal{S})$ of order $4 r$ with $r>1$ and $r \mid\left(q \pm 2 q_{0}+1\right)$ is conjugate in $\operatorname{Aut}(\mathcal{S})$ to a subgroup of $N_{\text {Aut }(\mathcal{S})}\left(\mathcal{S}_{ \pm 1}\right)$ (which has order $\left.4\left(q \pm 2 q_{0}+1\right)\right)$.

TheOREM 6.11. Let $\mathcal{U}$ be a subgroup of $\operatorname{Aut}(\mathcal{S})$ of order $4 r$ with $r>1$ a divisor of $q \pm 2 q_{0}+1$. Then the genus $\widetilde{g}$ of the quotient curve $\widetilde{\mathcal{S}}=\mathcal{S} / \mathcal{U}$ is given by

$$
\widetilde{g}= \begin{cases}\frac{q_{0}-1}{4}\left(\frac{q+2 q_{0}+1}{r}-1\right) & \text { for } r \mid\left(q+2 q_{0}+1\right), \\ \frac{q_{0}+1}{4}\left(\frac{q-2 q_{0}+1}{r}-1\right) & \text { for } r \mid\left(q-2 q_{0}+1\right) .\end{cases}
$$

Proof. The subgroup $\mathcal{U}$ comprises $r$ elements of odd order together with the same number of elements of order 2 and $2 r$ elements of order 4 . By (4.2) the degree of the ramification divisor of the natural map $\mathcal{S} \rightarrow \widetilde{\mathcal{S}}$ is $\left(2 q_{0}+2\right) r+$ $(2 r) 2+\widetilde{R}$, where $\widetilde{R}=0$ if $r \mid\left(q+2 q_{0}+1\right)$ and $\widetilde{R}=4(r-1)$ if $r \mid\left(q-2 q_{0}+1\right)$. Now the assertion follows by the Riemann-Hurwitz genus formula. 
Type VI. Let $q_{0}=2^{s}$. Set $\widetilde{q}:=2^{2 \widetilde{s}+1}$ with $\widetilde{s}$ a divisor of $s$ such that $2 \widetilde{s}+1$ divides $2 s+1$. This is an arithmetical (necessary) condition in order that $\operatorname{Aut}(\mathcal{S})$ contains a subgroup $\mathcal{U}$ isomorphic to $\mathcal{S} z(\widetilde{q})$.

THEOREM 6.12. With the notation above, the genus of $\widetilde{\mathcal{S}}=\mathcal{S} / \mathcal{U}$ is given by

$$
\widetilde{g}=\frac{q_{0}(q-1)-1+\left(\widetilde{q}^{2}+1\right) \widetilde{q}^{2}(\widetilde{q}-1)+\Delta}{\left(\widetilde{q}^{2}+1\right) \widetilde{q}^{2}(\widetilde{q}-1)},
$$

where

$$
\begin{aligned}
\Delta:= & \left(\widetilde{q}^{2}+1\right)\left[\left(2 q_{0}+2\right)(\widetilde{q}-1)+2 \widetilde{q}(\widetilde{q}-1)\right]+\widetilde{q}^{2}\left(\widetilde{q}^{2}+1\right)(\widetilde{q}-2) \\
& +\widetilde{q}^{2}\left(\widetilde{q}+2 \widetilde{q}_{0}+1\right)(\widetilde{q}-1)\left(\widetilde{q}-2 \widetilde{q}_{0}\right) .
\end{aligned}
$$

Proof. It is straightforward to see that $\mathcal{U}$ has $\left(\bar{q}^{2}+1\right)(\bar{q}-1)$ elements of order 2 , and $\left(\bar{q}^{2}+1\right)\left(\bar{q}^{2}-\bar{q}\right)$ elements of order 4 . Furthermore, $\mathcal{U}$ has $\frac{1}{2} \bar{q}^{2}\left(\bar{q}^{2}+1\right)$ subgroups of order $\bar{q}-1$. Also, $\mathcal{U}$ has $\frac{1}{4} \bar{q}^{2}\left(\bar{q}+2 \bar{q}_{0}+1\right)(\bar{q}-1)$ subgroups of order $\bar{q}-2 \bar{q}_{0}+1$. Finally, $\mathcal{U}$ has $\frac{1}{4} \bar{q}^{2}\left(\bar{q}-2 \bar{q}_{0}+1\right)(q-1)$ subgroups of order $\bar{q}+2 \bar{q}_{0}+1$. Thus by (4.2) the degree of the ramification divisor of $\mathcal{S} \rightarrow \widetilde{\mathcal{S}}$ equals

$$
\begin{aligned}
\left(\bar{q}^{2}+1\right)\left[\left(2 q_{0}+2\right)(\bar{q}-1)+2 \bar{q}(\bar{q}-1)\right] & +\bar{q}^{2}\left(\bar{q}^{2}+1\right)(\bar{q}-2) \\
& +\bar{q}^{2}\left(\bar{q}+2 \bar{q}_{0}+1\right)(\bar{q}-1)\left(\bar{q}-2 \bar{q}_{0}\right),
\end{aligned}
$$

whence the assertion follows by the Riemann-Hurwitz genus formula.

7. On curves with many rational points. Let $N_{q}(g)$ be the maximum number of $K$-rational points that a curve over $K$ of genus $g>0$ can have. Our references for this section are [8] and [9].

The study of the function $N_{q}(g)$ is strongly motivated by applications, as already mentioned in the introduction; in particular, good Goppa geometric codes have been constructed from the Suzuki curve by Matthews [24]. In general, no closed formula for $N_{q}(g)$ is known. The study of $N_{q}(g)$ viewed as a function of $g$ was initiated by Serre [29]. He was able to compute $N_{q}(1)$ and $N_{q}(2)$.

For any non-negative integer $g$,

$$
a_{q}(g) \leq N_{q}(g) \leq b_{q}(g) \leq q+2 g \sqrt{q}+1
$$

where $a_{q}(g)$ is the number of $K$-rational points of a specific curve over $K$ of genus $g$, and $b_{q}(g)$ is a theoretical upper bound. One often takes $b_{q}(g)$ as the smallest of three numbers: Serre's bound, Ihara's bound (cf. $[9$, p. 1]), and the one obtained via Explicit Formulas (see e.g. [31, V.3.4]).

We shall investigate the left hand side inequality in (7.1) with $q=2 q_{0}^{2}$, and the genus $g=\widetilde{g}$ of a quotient curve $\widetilde{\mathcal{S}}$ of the Suzuki curve $\mathcal{S}$. Note that by $(3.1), \# \widetilde{\mathcal{S}}(K)=q+2 q_{0} \widetilde{g}+1 \geq\lfloor b / \sqrt{2}\rfloor$; thus we might expect 
"many" $K$-rational points on the curve $\widetilde{\mathcal{S}}$ (this is the case when $q \leq 128$ and $\widetilde{g} \leq 50$ according to $[9$, Sect. 1$])$. We only consider the cases $q_{0}=2$, $q_{0}=4, q_{0}=8, \widetilde{g} \leq 50$ so that we can compare the number of rational points of the curves studied in this paper with the entries of van der Geer and van der Vlugt tables [9]; we remark that the tables are updated periodically.

1. $q_{0}=2$. Here $\# \widetilde{\mathcal{S}}(K)=9+4 \widetilde{g}$. We have the following data:

\begin{tabular}{c|ccccc}
\hline Reference & Cor. 6.5 & Thm. 4.1 & Cor. 6.5 & Cor. 6.5 & Cor. 6.5 \\
$\widetilde{g}$ & 1 & 2 & 3 & 6 & 14 \\
$N_{8}(\widetilde{g})$ & 14 & 18 & 24 & $33-35$ & 65 \\
$\# \widetilde{\mathcal{S}}(K)$ & 13 & 17 & 21 & $\mathbf{3 3}$ & $\mathbf{6 5}$ \\
\hline
\end{tabular}

The curve of genus 2 almost attains $N_{8}(2)$; it is given by

$$
V^{2}+V=\frac{(1+U)^{4} U^{7}}{f(U)^{2}},
$$

where $f(U)=U^{3}+U^{2}+1$ (Remark 4.7). It would be interesting to find an explicit equation of a plane model of a curve realizing $N_{8}(2)$. However, this appears to be out of reach. The cases $\widetilde{g}=6,14$ were already noticed by Stichtenoth [30, p. 205].

2. $q_{0}=4$. Here $\# \widetilde{\mathcal{S}}(K)=33+8 \widetilde{g}$. We have the following data:

\begin{tabular}{cccc}
\hline Reference & $\widetilde{g}$ & $N_{32}(\widetilde{g})$ & $\# \widetilde{\mathcal{S}}(K)$ \\
\hline Cor. 6.5 & 1 & 44 & 41 \\
Cor. 6.5 & 2 & 53 & 49 \\
Cor. 6.5 & 3 & 64 & 57 \\
Thm. 4.1 & 4 & $71-75$ & 65 \\
Thm. 6.11 & 5 & $83-86$ & 73 \\
Cor. 6.5 & 6 & $86-97$ & 81 \\
Thm. 6.10(2) & 10 & $\ldots$ & $\mathbf{1 1 3}$ \\
Cor. 6.5 & 12 & $129-163$ & $\mathbf{1 2 9}$ \\
Cor. 6.5 & 14 & $146-185$ & 145 \\
Thm. 5.1(2) & 24 & $\ldots$ & $\mathbf{2 2 5}$ \\
Cor. 6.5 & 28 & $257-298$ & $\mathbf{2 5 7}$ \\
Cor. 6.5 & 30 & $273-313$ & $\mathbf{2 7 3}$ \\
\hline
\end{tabular}

We have $113 \leq N_{32}(10) \leq 143$, where the upper bound is obtained from Serre's bound. The equation of $\widetilde{\mathcal{S}}$ is given by

$$
b f(V)=\left(U^{5}+U^{3} V^{5}+U V^{10}\right)\left(V^{3}+V^{7}\right),
$$


where $b=\lambda^{4}+\lambda^{3}+\lambda^{-4}+\lambda^{-3}$ with $\lambda \in \mathbb{F}_{32^{4}}$ of order 25 , and $f(V)=$ $1+V^{2}(1+V)+V^{9}(1+V)^{2}$. The cases $\widetilde{g}=12, \widetilde{g}=28$ and $\widetilde{g}=30$ were already noticed by van der Geer and van der Vlugt [11] via fiber products of certain Artin-Schreier curves. We do not know if such curves are isomorphic to the corresponding quotient curves $\widetilde{\mathcal{S}}$ here. The curve of genus $\widetilde{g}=14$ almost attains $N_{32}(14)$. We have $225 \leq N_{32}(24) \leq 245$, where the upper bound is obtained from Ihara's bound; a plane model of $\widetilde{\mathcal{S}}$ is given by

$$
b V^{5} f(U)=\left(U^{3}+U^{7}\right)\left(U^{25}+V^{10}\right),
$$

where $b$ and $f(U)$ are as above.

3. $q_{0}=8$. Here $\# \widetilde{\mathcal{S}}(K)=129+16 \widetilde{g}$. We have the following data:

\begin{tabular}{cccc}
\hline Reference & $\widetilde{g}$ & $N_{128}(\widetilde{g})$ & $\# \widetilde{\mathcal{S}}(K)$ \\
\hline Cor. 6.5 & 1 & 150 & 145 \\
Cor. 6.5 & 2 & 172 & 161 \\
Cor. 6.5 & 3 & 192 & 177 \\
Cor. 6.5 & 4 & $215-217$ & 193 \\
Cor. 6.5 & 6 & $243-261$ & 225 \\
Thm. 6.11 & 7 & $258-283$ & 241 \\
Cor. 6.5 & 8 & $257-305$ & $\mathbf{2 5 7}$ \\
Cor. 6.5 & 12 & $321-393$ & $\mathbf{3 2 1}$ \\
Thm. 6.10(1) & 14 & $353-437$ & $\mathbf{3 5 3}$ \\
Thm. 5.1(2) & 24 & $513-657$ & $\mathbf{5 1 3}$ \\
Cor. 6.5 & 28 & $577-745$ & $\mathbf{5 7 7}$ \\
Cor. 6.5 & 30 & $609-789$ & $\mathbf{6 0 9}$ \\
Thm. 5.1(1) & 36 & $\ldots$ & $\mathbf{7 0 5}$ \\
Thm. 6.11 & 49 & $\ldots$ & $\mathbf{9 1 3}$ \\
\hline
\end{tabular}

For $\widetilde{g}=8$, the existence of a curve with $257 K$-rational points was pointed out by Wirtz [34]. We find that $\widetilde{\mathcal{S}}$ is hyperelliptic and it is defined by (cf. Remark 4.7)

$$
V^{2}+V=\frac{(1+U)^{16} U^{127}}{f(U)^{2}}
$$

where $f(U)=1+U^{8}(1+U)+U^{25}(1+U)^{2}+U^{59}(1+U)^{4}$. We do not know whether $\widetilde{\mathcal{S}}$ is isomorphic to Wirtz's curve. For $\widetilde{g}=12,14,24,28,30$, the existence problem was solved affirmatively by van der Geer and van der Vlugt [11], [10]. Again, it is still unknown to us whether such curves are isomorphic to the corresponding $\widetilde{\mathcal{S}}$. Furthermore, $705 \leq N_{128}(36) \leq 921$; here the upper bound follows from Serre's bound. A plane equation for $\widetilde{\mathcal{S}}$ is 
given by

$$
V^{5} \tilde{f}(U)=U^{145}+V^{10}
$$

where $\tilde{f}(U)=1+U^{8}(1+U)+U^{16}(1+U)^{10}+U^{32}(1+U)^{28}+U^{64}$. Finally, $913 \leq N_{128}(49) \leq 1207$ where, again, the upper bound follows from Serre's bound. Unfortunately in this case we do not have an explicit plane model for $\widetilde{\mathcal{S}}$.

Acknowledgements. M. Giulietti and G. Korchmáros were supported by the Italian Ministry MIUR, project Strutture Geometriche, Combinatoria e loro applicazioni, PRIN 2001-2002, and by GNSAGA. The work of F. Torres was supported by the "Secretaria de Estado de Educación y Universidades del Ministerio de Educación, Cultura y Deportes de España" (SB2000-0225), CNPq-Brazil (306676/03-6) and PRONEX (66.2408/96-9).

\section{References}

[1] Y. Aubry and M. Perret, Divisibility of zeta functions of curves in a covering, Arch. Math. (Basel) 82 (2004), 205-213.

[2] E. Çakçak and F. Özbudak, Subfields of the function field of the Deligne-Lusztig curve of Ree type, Acta Arith. 115 (2004), 133-180.

[3] A. Cossidente, G. Korchmáros and F. Torres, On curves covered by the Hermitian curve, J. Algebra 216 (1999), 56-76.

[4] - - - - C Curves of large genus covered by the Hermitian curve, Comm. Algebra 28 (2000), 4707-4728.

[5] P. Deligne and G. Lusztig, Representations of reductive groups over finite fields, Ann. of Math. 103 (1976), 103-161.

[6] R. Fuhrmann and F. Torres, On Weierstrass points and optimal curves, Rend. Circ. Mat. Palermo. Suppl. 51 (1998), 25-46.

[7] A. Garcia, H. Stichtenoth and C. P. Xing, On subfields of the Hermitian function field, Compos. Math. 120 (2000), 137-170.

[8] G. van der Geer, Error-correcting codes and curves over finite fields, in: Mathematics Unlimited-2001 and Beyond, B. Engquist and W. Schmid (eds.), Springer, 2001, $1115-1138$.

[9] G. van der Geer and M. van der Vlugt, Tables of curves with many points, February 29, 2004; http://www.wins.uva.nl/ geer.

[10] - - -, Quadratic forms, generalized Hamming weights of codes and curves with many points, J. Number Theory 59 (1996), 20-36.

[11] - - -, Curves over finite fields of characteristic 2 with many rational points, C. R. Acad. Sci. Paris Sér. I 317 (1993), 593-597.

[12] V. D. Goppa, Geometry and Codes, Math. Appl. 24, Kluwer, Dordrecht, 1988.

[13] D. Gorenstein, Finite Groups, Chelsea, New York, 1980.

[14] J. P. Hansen, Deligne-Lusztig varieties and group codes, in: Lecture Notes in Math. 1518, Springer, 1992, 63-81.

[15] J. P. Hansen and J. P. Pedersen, Automorphism groups of Ree type, Deligne-Lusztig curves and function fields, J. Reine Angew. Math. 440 (1993), 99-109.

[16] J. P. Hansen and H. Stichtenoth, Group codes on certain algebraic curves with many rational points, Appl. Algebra Engrg. Comm. Comput. 1 (1990), 67-77. 
[17] R. Hartshorne, Algebraic Geometry, Grad. Texts in Math. 52, Springer, New York, 1977.

[18] H. W. Henn, Funktionenkörper mit großer Automorphismengruppe, J. Reine Angew. Math. 302 (1978), 96-115.

[19] J. W. P. Hirschfeld, Projective Geometries over Finite Fields, 2nd ed., Oxford Univ. Press, Oxford, 1998.

[20] B. Huppert and N. Blackburn, Finite Groups III, Springer, 1982.

[21] G. Lachaud, Sommes d'Eisenstein et nombre de points de certaines courbes algébriques sur les corps finis, C. R. Acad. Sci. Paris Sér. I 305 (1987), 729-732.

[22] R. Lidl and H. Niederreiter, Finite Fields, Cambridge Univ. Press, 1987.

[23] H. Lüneburg, Translation Planes, Springer, 1980.

[24] G. L. Matthews, Codes from the Suzuki function field, IEEE Trans. Inform. Theory 50 (2004), 3298-3302.

[25] C. J. Moreno, Algebraic Curves over Finite Fields, Cambridge Tracts in Math. 97, Cambridge Univ. Press, 1991.

[26] D. Mumford, Abelian Varieties, Tata Inst. Fund. Res. Stud. Math. 5, Oxford Univ. Press, Bombay, 1994.

[27] J. P. Pedersen, A function field related to the Ree group, in: Lecture Notes in Math. 1518, Springer, 1992, 122-131.

[28] H. G. Rück and H. Stichtenoth, A characterization of Hermitian function fields over finite fields, J. Reine Angew. Math. 457 (1994), 185-188.

[29] J. P. Serre, Sur le nombre des points rationnels d'une courbe algébrique sur un corps fini, C. R. Acad. Sci. Paris Sér I 296 (1983), 397-402.

[30] H. Stichtenoth, Algebraic-geometric codes associated to Artin-Schreier extensions of $\mathbb{F}_{q}(z)$, in: Proc. 2nd Int. Workshop on Alg. and Comb. Coding Theory, Leningrad, 1990, 203-206.

[31] -, Algebraic Function Fields and Codes, Springer, Berlin, 1993.

[32] K. O. Stöhr and J. F. Voloch, Weierstrass points and curves over finite fields, Proc. London Math. Soc. (3) 52 (1986), 1-19.

[33] J. Tits, Ovoïdes et groupes de Suzuki, Arch. Math. (Basel) 13 (1962), 187-198.

[34] M. Wirtz, Konstruktion und Tabellen linearer Codes, Westfälische Wilhelms-Univ. Münster, 1991.

Dipartimento di Matematica e Informatica

Università di Perugia

Dipartimento di Matematica 06123 Perugia, Italy

Università della Basilicata

E-mail: giuliet@dipmat.unipg.it

Campus Universitario Contrada Macchia Romana 85100 Potenza, Italy

IMECC-UNICAMP

E-mail: korchmaros@unibas.it

Cx.P. 6065

13083-970, Campinas SP, Brazil

E-mail: ftorres@ime.unicamp.br

Received on 8.6.2005

and in revised form on 6.2.2006 